\title{
Hamilton-Jacobi-Bellman equations with fast gradient-dependence
}

\author{
F. RAMPAZZO \& C. SARTORI
}

ABSTRACT. We investigate existence, uniqueness, and regularity properties for a class of $\mathrm{H}-\mathrm{J}-\mathrm{B}$ equations arising in non-linear control problems with unbounded controls. These equations involve Hamiltonians which are superlinear in the adjoint variable, and they have been already studied in the case when the growth in the adjoint variable is, in a sense, uniform with respect to the state variable. For instance, this is the case of the linear-quadratic problem. On the contrary, our results concern Hamiltonians that are superlinear in the adjoint variable, possibly not uniformly with respect to the state variable. Actually, this is the general situation one has to deal with when considering optimal control problems with a nonlinear dynamics (e.g. by slightly perturbing the linear quadratic problem). We also investigate situations where the fast growth of the Hamiltonian in the adjoint variable degenerates into a very discontinuity. Such Hamiltonians arise quite naturally in those optimal control problems where, roughly speaking, the dynamics and the cost display the same growth in the control variable.

\section{IntRoduction AND PRELIMINARIES}

Among the hypotheses under which a Cauchy problem of the form

$$
\begin{cases}-u_{t}+\mathcal{H}\left(t, x, u_{x}\right)=0, & (t, x) \in] 0, T\left[\times \mathbb{R}^{n}\right. \\ u(T, x)=g(x) & x \in \mathbb{R}^{n}\end{cases}
$$

is proved to admit at most one viscosity solution, the following condition on the Hamiltonian $\mathcal{H}$ plays a crucial role: 
For every $R>0$ there exists a modulus $\omega_{R}$, decreasing with $R$, such that

$$
\left|\mathcal{H}\left(t, x, \frac{x-y}{\varepsilon}\right)-\mathcal{H}\left(t, y, \frac{x-y}{\varepsilon}\right)\right| \leq \omega_{R}\left(|x-y|+\frac{|x-y|^{2}}{\varepsilon}\right)
$$

for all $\varepsilon>0,(x, y) \in B[0 ; R]$, and $t \in[0, T]$ (where $B[z ; \rho]$ denotes the closed ball of center $z$ and radius $\rho$ ).

Hypothesis (1.2) is verified if, for instance, $\mathcal{H}$ is Lipschitz continuous with respect to $x$ and grows at most linearly in the variable $u_{x}$. This is certainly true when

$$
\mathcal{H}\left(t, x, u_{x}\right) \doteq \sup _{c \in C}\left\{-\left\langle u_{x}, f(t, x, c)\right\rangle-\ell(t, x, c)\right\}
$$

that is, (1.1) is a Bellman equation-provided the data $f$ and $\ell$ are Lipschitz continuous in $x$ and $C$ is a compact subset of $\mathbb{R}^{m}$.

However, if $C$ is unbounded, the Hamiltonian $\mathcal{H}$ happens to be superlinear in $u_{x}$. In this event, in order to verify (1.2) one needs that the dependence of $\mathcal{H}$ on $u_{x}$ is uniform with respect to $x$. For instance, the Hamiltonian

$$
\mathcal{H}_{1}=u_{x}^{2}-x^{2}
$$

which corresponds to the case when $f=c$ and $l=x^{2}+c^{2} / 4$ (the linear quadratic problem), verifies (1.2). Instead, if $f=x c$, and $l=x^{2}+c^{2} / 4$, the Hamiltonian $\mathcal{H}$ reduces to

$$
\mathcal{H}_{2}=x^{2} u_{x}^{2}-x^{2}
$$

and there is no choice of the modulus $\omega$ for which (1.2) holds. Let us remark that the uniqueness problem for a class of Hamiltonians corresponding to an unbounded set $C$ has been recently addressed e.g. by P. Cannarsa and G. Da Prato [11], M. Bardi and F. Da Lio [3], H. Ishii [17], and W. McEneaney [19]. (See also W.Fleming and H.M. Soner [15], P. Soravia [25] for related questions, and A. Bensoussan [9] for the linear-quadratic case). Although a Hamiltonian like $\mathcal{H}_{2}$ does not agree with the hypotheses assumed in these papers, it fits the hypotheses made here (see $\left(A_{1}\right)-\left(A_{5}\right)$ below).

In the present paper we consider a Hamiltonian $\mathcal{H}$ as in (1.3), with an $u n$ bounded control set $C \subseteq \mathbb{R}^{m}$, and functions $f(t, x, \cdot)$ and $\ell(t, x, \cdot)$ growing as $|c|^{\alpha},|c|^{\beta}$, respectively, with $(1 \leq \alpha \leq \beta)$. The above examples show that, unless additional hypotheses are made on $f$ and $\ell$, condition (1.2) is not verified.

More precisely, let $\alpha, \beta$ be numbers satisfying $1 \leq \alpha \leq \beta$, and let $C$ be a closed (possibly unbounded) subset of $\mathbb{R}^{m}$. We assume the following hypotheses 
on the data $f, \ell$, and $g$ :

$\left(\mathrm{A}_{1}\right) f:[0, T] \times \mathbb{R}^{n} \times \mathbb{R}^{m} \rightarrow \mathbb{R}^{n}$ is continuous and, for every compact subset $Q \subset[0, T] \times \mathbb{R}^{n}$ there exists a positive constant $L$ and a modulus $\omega_{f}$ verifying

$$
\left|f\left(t_{1}, x_{1}, c\right)-f\left(t_{2}, x_{2}, c\right)\right| \leq\left(1+|c|^{\alpha}\right)\left(L\left|x_{1}-x_{2}\right|+\omega_{f}\left(\left|t_{1}-t_{2}\right|\right),\right.
$$

for all $\left(t_{1}, x_{1}, c\right),\left(t_{2}, x_{2}, c\right) \in Q \times \mathbb{R}^{m}$ (by modulus we mean a positive, nondecreasing function, null and continuous at zero).

$\left(\mathrm{A}_{2}\right) \quad \ell:[0, T] \times \mathbb{R}^{n} \times \mathbb{R}^{m} \rightarrow \mathbb{R}$ is continuous and, for every compact subset $Q \subset[0, T] \times \mathbb{R}^{n}$, there is a modulus $\omega_{\ell}$ satisfying

$$
\left|\ell\left(t_{1}, x_{1}, c\right)-\ell\left(t_{2}, x_{2}, c\right)\right| \leq\left(1+|c|^{\beta}\right) \omega_{\ell}\left(\left|\left(t_{1}, x_{1}\right)-\left(t_{2}, x_{2}\right)\right|\right)
$$

for every $\left(t_{1}, x_{1}, c\right),\left(t_{2}, x_{2}, c\right) \in Q \times \mathbb{R}^{m}$.

$\left(\mathrm{A}_{3}\right)$ There exist non-negative constants $M_{1}$ and $M_{2}$ such that

$$
|f(t, x, c)| \leq M_{1}\left(1+|c|^{\alpha}\right)(1+|x|)+M_{2}\left(1+|c|^{\alpha}\right)
$$

for every $(t, x, c) \in[0, T] \times \mathbb{R}^{n} \times \mathbb{R}^{m}$.

$\left(\mathrm{A}_{4}\right)$ There exist positive constants $\ell_{0}$ and $\ell_{1}$ such that the following coercivity condition

$$
\ell(t, x, c) \geq \ell_{0}|c|^{\beta}-\ell_{1},
$$

is verified for every $(t, x, c) \in[0, T] \times \mathbb{R}^{n} \times \mathbb{R}^{m}$.

$\left(\mathrm{A}_{5}\right)$ The map $g: \mathbb{R}^{n} \rightarrow \mathbb{R}$ is continuous and bounded below.

We assume also a condition of regularity of $f$ and $\ell$ at infinity in the variable $c$. Precisely, we posit the existence of continuous functions $f^{\infty}$ and $\ell^{\infty}$, the recessions functions of $f$ and $\ell$, respectively, verifying

$$
\lim _{r \rightarrow 0} r^{\beta} f\left(t, x, r^{-1} w\right) \doteq f^{\infty}(t, x, w)
$$

and

$$
\lim _{r \rightarrow 0} r^{\beta} \ell\left(t, x, r^{-1} w\right) \doteq \ell^{\infty}(t, x, w),
$$

on compact sets of $[0, T] \times \mathbb{R}^{n} \times \mathbb{R}^{m}$ (e.g., if $f(t, x, c)=f_{0}(t, x)+f_{1}(t, x)|c|+$ $f_{2}(t, x)|c|^{2}$ and $\beta=2$, then $\left.f^{\infty}(t, x, w)=f_{2}(t, x)|w|^{2}\right)$.

Under the above hypotheses-which of course can be weakened in several directions, see e.g. Remark 2.5-in Section 2 we prove a comparison result for (1.1) which, in particular, implies that the (viscosity) solution of (1.1) is unique and 
continuous. These results are obtained by showing that problem (1.1) is equivalent to a more regular problem, which involves a Hamiltonian which grows at most linearly in the gradient variable.

Let us remark that the case when $\alpha=\beta$ is a special one, for in general the Hamiltonian is discontinuous. This leads us to utilize an extended version of the notion of viscosity solution introduced by $\mathrm{H}$. Ishii in [16]. Moreover when the Hamiltonian is discontinuous, even the recent results in [17] do not apply. A further peculiarity of the case when $\alpha=\beta$ consists in the fact that, in order to get existence (and uniqueness) of the solution, the boundary condition in (1.1) has to be replaced with a more general condition $\left(\right.$ see $\left(\mathrm{BC}_{m}\right)$ in Section 2. This condition keeps track of the possible occurrence of final jumps of the optimal trajectories of the underlying control problem. However, provided a suitable quantitative assumption is imposed on $f, \ell$ and $g$, (see (NFJ) in Section 3, existence (and uniqueness) for the original Cauchy problem (1.1) can be recovered also when $\alpha=\beta$. Section 3 is devoted to embed the underlying control problem into an auxiliary, more regular, control problem. For this purpose we introduce a reparameterization of time based on the coercivity exponent $\beta$ involved in assumption $\left(\mathrm{A}_{4}\right)$. Section 4 is devoted to the study of regularity properties of the unique solution of (1.1). Let us first point out that even in the case $\alpha=\beta$, despite the fact that the Hamiltonian happens to be discontinuous, the solution is continuous. However, passing from $\alpha<\beta$ to $\alpha=\beta$ causes a loss of regularity in the $t$ dependence of the solution to (1.1). Again, this can be explained with an argument based on characteristics, namely the optimal trajectories of the underlying control problem. Indeed the latter are continuous when $\alpha<\beta$, while when $\alpha=\beta$ they may contain jumps (to be interpreted as limits of minimizing sequences). This same argument also explains why there is no discrepancy between the case $\alpha<\beta$ and the case $\alpha=\beta$ when a suitable quantitative condition on the data (see (NJ) in Section 4, which prevents from jumps, is in force. The paper is concluded by an Appendix, where a simplifying hypothesis assumed throughout the paper-namely the fact that the control set of the underlying control problem is a cone-is shown to be inessential.

Let us observe that though we have restricted our attention to the Cauchy problem (1.1) — which is connected to the study of a Bolza control problemextensions to (e.g. stationary) Bellman equations arising from different control problems are quite plausible. Generalizations to cases where $\mathcal{H}$ is no longer convex will be the matter of future work. Yet, let us mention that for $\alpha=\beta=1$, situations when the supremum in (1.3) is replaced with a inf sup (or a sup inf) have been already investigated in [8] and [23].

\section{EXISTENCE, UNIQUENESS AND CONTINUITY OF SOLUTIONS}


Let us recall Ishii's extension of the definition of viscosity solution of a HamiltonJacobi equation [16]. This definition reduces to the classical one (see e.g. [13] and [12]) as soon as the solution and the Hamiltonian are continuous.

Given a function $F: \mathcal{Q} \rightarrow \mathbb{R}, \mathcal{Q} \subseteq \mathbb{R}^{k}$, the upper and lower semicontinuous envelopes are defined by

$$
\begin{gathered}
F^{*}(x) \doteq \lim _{r \rightarrow 0^{+}} \sup \{F(y): y \in \mathcal{Q},|x-y| \leq r\}, \\
F_{*}(x) \doteq \lim _{r \rightarrow 0^{+}} \inf \{F(y): y \in \mathcal{Q},|x-y| \leq r\}, \quad x \in \overline{\mathcal{Q}},
\end{gathered}
$$

respectively. Of course, $F^{*}$ is upper semicontinuous and $F_{*}$ is lower semicontinuous.

Definition 2.1. Let $E$ be a subset of $\mathbb{R}^{s}$ and let $G$ be a real map, the Hamiltonian, defined on $E \times \mathbb{R} \times \mathbb{R}^{s}$. An upper [resp. lower]-semicontinuous function $u$ is a viscosity subsolution [resp. supersolution] of

$$
G\left(y, u, u_{y}\right)=0
$$

at $y \in E$ if for every $\varphi \in C^{1}\left(\mathbb{R}^{s}\right)$ such that $y$ is a local maximum [resp. minimum] point of $u-\varphi$ on $E$, one has

$$
G_{*}\left(y, \varphi(y), \varphi_{y}(y)\right) \leq 0,
$$

[respectively

$$
\left.G^{*}\left(y, \varphi(y), \varphi_{y}(y)\right) \geq 0 .\right]
$$

A function $u$ is a viscosity solution of (2.1) at $y \in E$ if $u^{*}$ is a viscosity subsolution at $y$ and $u_{*}$ is a viscosity supersolution at $y$.

Let us consider a Hamilton-Jacobi-Bellman equation of the form

$$
H\left(t, x, u_{t}, u_{x}\right)=0
$$

where $u$ denotes a real function defined on $[0, T] \times \mathbb{R}^{n}, u_{t}$ and $u_{x}$ denote the gradients of $u$ with respect to the variables $t$ and $x$ respectively, and

$$
H\left(t, x, p_{0}, p\right) \doteq \sup _{c \in C}\left\{-p_{0}-\langle p, f(t, x, c)\rangle-\ell(t, x, c)\right\} .
$$

(Note that (HJ) coincides with (1.3). The change of notation is justified by the introduction of an auxiliary Hamiltonian (see Section 3), where $t$ is regarded as a state variable.) 
The control set $C$ that appears in the definition of $H$ is a (possibly unbounded) subset of $\mathbb{R}^{m}$. For the sake of simplicity, we assume that $C$ is a closed cone of $\mathbb{R}^{m}$, that is, a closed subset invariant by multiplication by non-negative numbers. However, this extra assumption is not crucial, and in the Appendix we shall outline the changes which are needed in order to address the general case.

Let us introduce two boundary value problems for the equation $(\mathrm{HJ})$.

Definition 2.2. A map $u:[0, T] \times \mathbb{R}^{n} \rightarrow \mathbb{R}$ is called a solution of the problem (HJ)-(BC) if $u$ is continuous on $\left(\{0\} \times \mathbb{R}^{n}\right) \cup\left(\{T\} \times \mathbb{R}^{n}\right), u$ is a viscosity solution of $(\mathrm{HJ})$ in $] 0, T\left[\times \mathbb{R}^{n}\right.$, and it satisfies the following Cauchy condition:

$$
u(T, x)=g(x) \quad \forall x \in \mathbb{R}^{n} .
$$

A map $u:[0, T] \times \mathbb{R}^{n} \rightarrow \mathbb{R}$ is called a solution of the problem $(\mathrm{HJ})-\left(\mathrm{BC}_{m}\right)$ if $u$ is continuous on $\left(\{0\} \times \mathbb{R}^{n}\right) \cup\left(\{T\} \times \mathbb{R}^{n}\right), u$ is a viscosity solution of $(\mathrm{HJ})$ in ] $0, T\left[\times \mathbb{R}^{n}\right.$, and it satisfies the following mixed type boundary condition:

$\left(\mathrm{BC}_{m}\right)$

$$
u(T, x) \leq g(x) \quad \forall x \in \mathbb{R}^{n}
$$

and

either $u(T, x)=g(x)$ or $u$ is a viscosity supersolution of $(\mathrm{HJ})$ at $(T, x)$.

Let us begin with two existence theorems which are straightforward consequences of Theorem 3.1 and 3.2 below (see Section 3). In Corollary 2.1 below we shall prove that the (existing) solution is unique and continuous.

Theorem 2.1. Let hypotheses $\left(\mathrm{A}_{1}\right)-\left(\mathrm{A}_{5}\right)$ be in force.

(i) Assume $\alpha<\beta$. Then there exists a solution of the boundary value problem $(\mathrm{HJ})-(\mathrm{BC})$.

(ii) Assume $\alpha=\beta$. Then there exists a solution of the boundary value problem $(\mathrm{HJ})-\left(\mathrm{BC}_{m}\right)$.

Proof. See Theorem 3.1.

Remark 2.1. When $\alpha=\beta$ the weakening of $(\mathrm{BC})$ into $\left(\mathrm{BC}_{m}\right)$ turns out to be crucial for the existence of a solution. For instance, consider the simple case where $f(t, x, c)=c, \ell(t, x, c)=|c|$, and $g(x)=L_{g} x$ with $L_{g} \geq 0, x$ and $c \in \mathbb{R}$, and 
$0 \leq t \leq 1$. The associated Hamilton-Jacobi equation turns out to be the following:

$$
-u_{t}+\sup _{c \in \mathbb{R}}\left\{-c u_{x}-|c|\right\}=0 .
$$

Notice that the left hand side of (2.2) is discontinuous in $u_{x}$, so the extended notion of viscosity solution given in Definition 2.1 has to be considered. It is easy to check that if $1 \leq L_{g}$ then the map $u(t, x)=|x|$ solves the boundary value problem (2.2)-( $\left(\mathrm{BC}_{m}\right)$. By the uniqueness result stated in Corollary (2.1) below, this is in fact the only solution of $(2.2)-\left(\mathrm{BC}_{m}\right)$. Since $(\mathrm{BC})$ is a special case of $\left(\mathrm{BC}_{m}\right)$, this implies that the Cauchy condition $u(1, x)=L_{g}|x|$ with $L_{g}>1$ does not allow any solution.

If the data satisfy the no-final-jump condition (2.3) below, (see Theorem 3.1) then we have existence for the Cauchy problem (HJ)-(BC) in the case $\alpha=\beta$ as well:

Theorem 2.2. Assume that $\alpha=\beta$ and that hypotheses $\left(\mathrm{A}_{1}\right)-\left(\mathrm{A}_{5}\right)$ are verified. Moreover, assume that the constant $M_{1}$ in condition $\left(\mathrm{A}_{3}\right)$ is equal to zero and that $g$ is Lipschitz continuous with constant $L_{g}$. If

$$
\ell_{0} \geq M_{2} L_{g}
$$

$\left(\right.$ see $\left(A_{4}\right)$ and $\left.\left(A_{3}\right)\right)$, then there exists a solution of the boundary value problem $(\mathrm{HJ})$ (BC).

Proof. See Theorem 3.2.

Remark 2.2. The no-final-jump condition (2.3) is optimal. Indeed in the simple example of the previous remark this condition reduces to

$$
1 \geq L_{g},
$$

while, as soon as $1<L_{g}$, no solution of $(\mathrm{HJ})$-(BC) exists.

The remaining part of this section is devoted to the proof of the following two results:

Theorem 2.3 (Comparison for $(\mathrm{HJ}))$. Assume $\left(\mathrm{A}_{1}\right)-\left(\mathrm{A}_{5}\right)$. Let $u_{1}:[0, T] \times$ $\mathbb{R}^{n} \rightarrow \mathbb{R}$ be an upper semicontinuous, bounded below, viscosity subsolution of $(\mathrm{HJ})$ in $] 0, T\left[\times \mathbb{R}^{n}\right.$, continuous on $\left(\{0\} \times \mathbb{R}^{n}\right) \cup\left(\{T\} \times \mathbb{R}^{n}\right)$. Let $u_{2}:[0, T] \times \mathbb{R}^{n} \rightarrow \mathbb{R}$ be a lower semicontinuous, bounded below, viscosity supersolution of $(\mathrm{HJ})$ in $[0, T[\times$ $\mathbb{R}^{n}$. Assume that for every $x \in \mathbb{R}^{n}$, one has and

either $u_{1}(T, x) \leq u_{2}(T, x)$

or $u_{2}$ is a viscosity supersolution of $(\mathrm{HJ})$ at $(T, x)$. 
Then

$$
u_{1}(t, x) \leq u_{2}(t, x) \quad \forall(t, x) \in[0, T] \times \mathbb{R}^{n} .
$$

Theorem 2.3 is a direct consequence of Theorems 2.4 and 2.5 below. Indeed, in Theorem 2.4 we state a comparison result for two related Cauchy problems involving the regularized equation

$$
H_{e}\left(t, x, u_{t}, u_{x}\right)=0 \text {, }
$$

where $H_{e}$ is introduced below. Secondly, in Theorem 2.5, we show that the sets of subsolutions and supersolutions of $(\mathrm{HJ})$ coincide with the sets of subsolutions and supersolutions of $\left(\mathrm{HJ}_{e}\right)$, respectively.

Corollary 2.1 (Uniqueness and continuity of the solution of $(\mathrm{HJ}))$. Assume $\left(\mathrm{A}_{1}\right)-\left(\mathrm{A}_{5}\right)$. Then, if $\alpha<\beta$ [resp. $\left.\alpha=\beta\right]$, problem $(\mathrm{HJ})-(\mathrm{BC})\left[\right.$ resp. $\left.(\mathrm{HJ})-\left(\mathrm{BC}_{m}\right)\right]$ admits only one, bounded below, viscosity solution. Moreover, this solution is continuous.

Proof. Suppose $v$ and $u$ are two solutions of (HJ)-(BC) [resp. of (HJ)$\left.\left(\mathrm{BC}_{m}\right)\right]$. Then $v^{*}$ and $u^{*}$ are viscosity subsolutions in $] 0, T\left[\times \mathbb{R}^{n}\right.$ and $v_{*}$ and $u_{*}$ are viscosity supersolutions in $] 0, T\left[\times \mathbb{R}^{n}\right.$ of $(\mathrm{HJ})-(\mathrm{BC})$ [resp. of $\left.(\mathrm{HJ})-\left(\mathrm{BC}_{m}\right)\right]$. Moreover, since $v$ and $u$ are continuous on $\{0\} \times \mathbb{R}^{n}$, by Lemma 2.1 and Theorem 2.5 below they are supersolutions of $(\mathrm{HJ})$ on $\{0\} \times \mathbb{R}^{n}$. Finally, since $v$ and $u$ are continuous on $\{T\} \times \mathbb{R}^{n}$, by Theorem 2.3 one has

$$
u^{*} \leq v_{*} \leq v^{*} \leq u_{*},
$$

which implies the theorem.

Remark 2.3. When $\alpha<\beta$ (the strictly coercive case), the Cauchy problem (HJ)-(BC) is trivially related to an optimal control problem where $f, \ell$ and $g$ are the dynamics, the running cost and the terminal cost, respectively (see the next section). When $\alpha=\beta,(\mathrm{HJ})-\left(\mathrm{BC}_{m}\right)$ is still related to an optimal control problem and the fact that $\left(\mathrm{BC}_{m}\right)$ involves also a supersolution condition is due to the possible occurrence of jumps of optimal trajectories. Finally, when condition (2.3) is in force such jumps are penalized, which yields a heuristic meaning of Theorem 2.2.

Remark 2.4. The boundary value problems $(\mathrm{HJ})-(\mathrm{BC})$ and $(\mathrm{HJ})-\left(\mathrm{BC}_{m}\right)$ do not satisfy the hypotheses assumed in [3] and [17], where questions similar to the ones studied here are addressed. In fact, in [17] and [3] hypothesis $\left(A_{1}\right)$ is replaced by the stronger hypotheses

$$
\left|f\left(x_{1}, c\right)-f\left(x_{2}, c\right)\right| \leq L\left|x_{1}-x_{2}\right|
$$


and

$$
\left\langle\left(f\left(x_{1}, c\right)-f\left(x_{2}, c\right)\right),\left(x_{1}-x_{2}\right)\right\rangle \leq L\left(x_{1}-x_{2}\right)^{2},
$$

respectively. Notice that both hypothesis (2.4) and hypothesis (2.5) (when also $\left(\mathrm{A}_{2}\right)$ and $\left(\mathrm{A}_{4}\right)$ are assumed) agree with the classical condition (1.2), while they do not fit, for example, dynamics like

$$
f(x, c)=f_{0}(x)+f_{1}(x) c .
$$

(Actually, [3] and [17] are mainly concerned with the unboundedness of the solution and do not address the problem of removing a condition like (1.2)). Instead, this kind of dynamics (and any dynamics with polynomial growth in $c$ ) is covered by our assumptions. Finally, in the case when $\alpha=\beta$, the Hamiltonian $H$ is in general discontinuous, so the results in [17] cannot be applied (even if hypothesis (2.4) is in force).

Remark 2.5. Of course, hypotheses $\left(\mathrm{A}_{1}\right)-\left(\mathrm{A}_{5}\right)$ are not the weakest possible. For instance, it is easy to check that all the results we present here remain true if one replaces $\left(A_{1}\right)$ and $\left(A_{4}\right)$ with hypotheses $\left(A_{1}^{\prime}\right)$ and $\left(A_{4}^{\prime}\right)$ below:

$\left(\mathrm{A}_{1}^{\prime}\right)$ For every compact subset $Q \subset[0, T] \times \mathbb{R}^{n}$ there exists $L>0$ such that

$$
\begin{aligned}
\langle(f(t, x, c)-f(t, y, c)) \cdot(x-y)\rangle \leq L\left(1+|c|^{\alpha}\right)|x-y|^{2}, & \\
& \forall(t, x),(t, y) \in Q, \quad \forall c \in \mathbb{R}^{m} .
\end{aligned}
$$

$\left(\mathrm{A}_{4}^{\prime}\right)$ There are positive constants $\ell_{0}$ and $\ell_{1}$ such that

$$
\ell(t, x, c) \geq \ell_{0}|c|^{\beta}-\ell_{1}|x|, \quad \forall(t, x, c) \in[0, T] \times \mathbb{R}^{n} \times \mathbb{R}^{m} .
$$

In order to state Theorems 2.4 and 2.5 below (of which Theorem 2.3 is a direct consequence), let us introduce the extended Hamiltonian

$$
\begin{aligned}
& H_{e}\left(t, x, p_{0}, p\right) \\
& \quad=\sup _{\left(w_{0}, w\right) \in\left(\left[0,+\infty[\times C) \cap S_{+}^{m}\right.\right.}\left\{-p_{0} w_{0}^{\beta}-\left\langle p, \bar{f}\left(t, x, w_{0}, w\right)\right\rangle-\bar{\ell}\left(t, x, w_{0}, w\right)\right\},
\end{aligned}
$$

where $S_{+}^{m} \doteq\left\{\left(w_{0}, w\right) \in\left[0,+\infty\left[\times \mathbb{R}^{m}:\left|\left(w_{0}, w\right)\right|=1\right\}\right.\right.$, and $\bar{f}$ and $\bar{\ell}$ are the continuous functions defined as follows for every $\left(t, x, w_{0}, w\right) \in[0, T] \times \mathbb{R}^{n} \times$ 
$[0,+\infty[\times C:$

$$
\bar{f}\left(t, x, w_{0}, w\right) \doteq \begin{cases}f\left(t, x, \frac{w}{w_{0}}\right) \cdot w_{0}^{\beta} & \text { if } w_{0} \neq 0, \\ f^{\infty}(t, x, w) & \text { if } w_{0}=0 \text { and } \alpha=\beta\end{cases}
$$

and

$$
\bar{\ell}\left(t, x, w_{0}, w\right) \doteq \begin{cases}\ell\left(t, x, \frac{w}{w_{0}}\right) \cdot w_{0}^{\beta} & \text { if } w_{0} \neq 0, \\ \ell^{\infty}(t, x, w) & \text { if } w_{0}=0 \text { and } \alpha=\beta .\end{cases}
$$

Let us remark that the introduction of the Hamiltonian $H_{e}$ is not a mere tool for the proof of Theorem 2.3. In fact, $H_{e}$ is the standard Hamiltonian of a spacetime optimal control problem obtained from the one underlying $(\mathrm{HJ})$ by means of a $\beta$-power reparameterization of time (see Section 3). The use of the reparameterized system seems particularly suitable in the case when $\alpha=\beta$, where limits of minimizing sequences of trajectories — which could be thought as the characteristic curves of $(\mathrm{HJ}$ — cannot merely be described in terms of discontinuous trajectories of the original system. Rather, these limits find a satisfactory description in terms of graph-completions, which are continuous paths in the space of graphs (see e.g. [20] for the case $\alpha=\beta=1$ ). Finally, since $H_{e}$ involves only bounded controls, this Hamiltonian appears quite adequate for questions of numerical interest (see e.g. [10] for the case $\alpha=\beta=1$ ).

Let us begin by stating some properties of the maps $\bar{f}$ and $\bar{\ell}$ which appear in the definition of $H_{e}$. These properties are obvious consequences of assumptions $\left(A_{1}\right)-\left(A_{4}\right)$.

\section{Proposition 2.1.}

(i) The functions $\bar{f}$ and $\bar{\ell}$ are continuous on $[0, T] \times \mathbb{R}^{n} \times\left[0,+\infty\left[\times \mathbb{R}^{m}\right.\right.$ and for every compact $Q \subset[0, T] \times \mathbb{R}^{n}$ we have

$\left(\mathrm{A}_{e_{1}}\right)$

$$
\begin{aligned}
& \left|\bar{f}\left(t_{1}, x_{1}, w_{0}, w\right)-\bar{f}\left(t_{2}, x_{2}, w_{0}, w\right)\right| \\
& \quad \leq\left(w_{0}^{\alpha}+|w|^{\alpha}\right) w_{0}^{\beta-\alpha}\left(L\left|x_{1}-x_{2}\right|+\omega_{f}\left(\left|t_{1}-t_{2}\right|\right)\right),
\end{aligned}
$$

and

$$
\begin{aligned}
& \left(\mathrm{A}_{e_{2}}\right) \quad\left|\bar{\ell}\left(t_{1}, x_{1}, w_{0}, w\right)-\bar{\ell}\left(t_{2}, x_{2}, w_{0}, w\right)\right| \\
& \leq\left(w_{0}^{\beta}+|w|^{\beta}\right) \omega_{\ell}\left(\left|\left(t_{1}, x_{1}\right)-\left(t_{2}, x_{2}\right)\right|\right), \\
& \forall\left(t_{1}, x_{1}, w_{0}, w\right),\left(t_{2}, x_{2}, w_{0}, w\right) \in[0, T] \times \mathbb{R}^{n} \times\left[0,+\infty\left[\times \mathbb{R}^{m}, \text { where } L,\right.\right.
\end{aligned}
$$


$\omega_{f}$ and $\omega_{\ell}$ are the same as in assumptions $\left(\mathrm{A}_{1}\right)$ and $\left(\mathrm{A}_{2}\right)$. Moreover,
$\left(\mathrm{A}_{e_{3}}\right)$

$$
\left|\bar{f}\left(t, x, w_{0}, w\right)\right| \leq\left(w_{0}^{\alpha}+|w|^{\alpha}\right) w_{0}^{\beta-\alpha}\left(M_{1}(1+|x|)+M_{2}\right),
$$

and

$\left(\mathrm{A}_{e_{4}}\right)$

$$
\bar{\ell}\left(t, x, w_{0}, w\right) \geq \ell_{0}|w|^{\beta}-\ell_{1}\left|w_{0}\right|^{\beta},
$$

$\forall\left(t, x, w_{0}, w\right) \in[0, T] \times \mathbb{R}^{n} \times\left[0,+\infty\left[\times \mathbb{R}^{m}\right.\right.$, where $M_{1}, M_{2}, \ell_{0}$ and $\ell_{1}$ are the same as in $\left(\mathrm{A}_{3}\right)$ and $\left(\mathrm{A}_{4}\right)$.

(ii) (Positive homogeneity in $\left.\left(w_{0}, w\right)\right)$. The map $\bar{f}$ and $\bar{\ell}$ are positively homogeneous of degree $\beta$ in $\left(w_{0}, w\right)$, that is,

$$
\bar{f}\left(t, x, r w_{0}, r w\right)=r^{\beta} \bar{f}\left(t, x, w_{0}, w\right)
$$

and

$$
\begin{aligned}
& \bar{\ell}\left(t, x, r w_{0}, r w\right)=r^{\beta} \bar{\ell}\left(t, x, w_{0}, w\right) \\
&\left.\forall r>0, \forall\left(t, x, w_{0}, w\right) \in[0, T] \times \mathbb{R}^{n} \times\right] 0,+\infty\left[\times \mathbb{R}^{m} .\right.
\end{aligned}
$$

Let us introduce two kinds of boundary condition for the equation $\left(\mathrm{HJ}_{e}\right)$ which are akin to those introduced in Definition 2.1 for the equation $(\mathrm{HJ})$.

Definition 2.3. A map $u:[0, T] \times \mathbb{R}^{n} \rightarrow \mathbb{R}$ is called a solution of the boundary value problem $\left(\mathrm{HJ}_{e}\right)-\left(\mathrm{BC}_{e}\right)$ if $u$ is continuous on $\left(\{0\} \times \mathbb{R}^{n}\right) \cup\left(\{T\} \times \mathbb{R}^{n}\right), u$ is a viscosity solution of

$$
H_{e}\left(t, x, u_{t}, u_{x}\right)=0,
$$

in $] 0, T\left[\times \mathbb{R}^{n}\right.$ and it satisfies the Cauchy condition:

$\left(\mathrm{BC}_{e}\right)$

$$
u(T, x)=g(x) \quad \forall x \in \mathbb{R}^{n} .
$$

A map $u:[0, T] \times \mathbb{R}^{n} \rightarrow \mathbb{R}$ is called a solution of the following boundary value problem $\left(\mathrm{HJ}_{e}\right)-\left(\mathrm{BC}_{e_{m}}\right)$ if $u$ is continuous on $\left(\{0\} \times \mathbb{R}^{n}\right) \cup\left(\{T\} \times \mathbb{R}^{n}\right)$, is a viscosity solution of $\left(\mathrm{HJ}_{e}\right)$ in $] 0, T\left[\times \mathbb{R}^{n}\right.$ and it satisfies the mixed type boundary condition:

$\left(\mathrm{BC}_{e_{m}}\right)$

$$
u(T, x) \leq g(x) \quad \forall x \in \mathbb{R}^{n}
$$

and

either $u(T, x)=g(x)$ or $u$ is a viscosity supersolution of $\left(\mathrm{HJ}_{e}\right)$ at $(T, x)$. 
Theorem 2.4 below concerns the comparison of viscosity subsolutions and supersolutions for the problems $\left(\mathrm{HJ}_{e}\right)-\left(\mathrm{BC}_{e}\right)$ and $\left(\mathrm{HJ}_{e}\right)-\left(\mathrm{BC}_{e_{m}}\right)$.

Theorem 2.4 (Comparison for $\left.\left(\mathrm{HJ}_{e}\right)\right)$. Assume $\left(\mathrm{A}_{1}\right)-\left(\mathrm{A}_{5}\right)$, with $\alpha \leq \beta$. Let $u_{1}:[0, T] \times \mathbb{R}^{n} \rightarrow \mathbb{R}$ be an upper semicontinuous, bounded below, viscosity subsolution of $\left(\mathrm{HJ}_{e}\right)$ in $] 0, T\left[\times \mathbb{R}^{n}\right.$, continuous on $\left(\{0\} \times \mathbb{R}^{n}\right) \cup\left(\{T\} \times \mathbb{R}^{n}\right)$. Let $u_{2}:[0, T] \times \mathbb{R}^{n} \rightarrow \mathbb{R}$ be a lower semicontinuous, bounded below, viscosity supersolution of $\left(\mathrm{HJ}_{e}\right)$ in $\left[0, T\left[\times \mathbb{R}^{n}\right.\right.$. For every $x \in \mathbb{R}^{n}$, assume that

$$
\begin{aligned}
& \text { either } u_{1}(T, x) \leq u_{2}(T, x) \text { or } u_{2} \text { is a viscosity supersolution of } \\
& \left(\mathrm{HJ}_{e}\right) \text { at }(T, x) .
\end{aligned}
$$

Then

$$
u_{1}(t, x) \leq u_{2}(t, x) \quad \forall(t, x) \in[0, T] \times \mathbb{R}^{n} .
$$

Proof. Let $G$ be a real number such that $u_{1}(t, x) \geq G$ and $u_{2}(t, x) \geq G$ for every $(t, x) \in[0, T] \times \mathbb{R}^{n}$. Let us consider the map $\Lambda$ defined by

$$
\Lambda(t, u) \doteq(1+t)\left(1-e^{-(u-G) \mu}\right),
$$

where $\mu=\left[(1+T) 2 \ell_{1}\right]^{-1}$, and consider the following Kružkov-type change of independent variables :

$$
\begin{aligned}
& v_{1}(t, x) \doteq \Lambda\left(t, u_{1}(t, x)\right) \\
& v_{2}(t, x) \doteq \Lambda\left(t, u_{2}(t, x)\right) .
\end{aligned}
$$

It is easy to check that $v_{1}$ [resp. $v_{2}$ ] is a viscosity subsolution [resp. supersolution] of the equation

$$
\begin{aligned}
\max _{\left(w_{0}, w\right) \in\left(\left[0,+\infty[\times C) \cap S_{+}^{m}\right.\right.}\left(v\left(\frac{w_{0}^{\beta}}{1+t}+\bar{\ell} \mu\right)-\right. & \left(\nabla_{t} v\right) w_{0}^{\beta} \\
& \left.-\left\langle\nabla_{x} v, \bar{f}\right\rangle-\bar{\ell}(1+t) \mu\right)=0
\end{aligned}
$$

in $] 0, T\left[\times \mathbb{R}^{n}\right.$ such that $v_{1}(T, x) \leq v_{2}(T, x)$ [resp. $v_{2}$ is a supersolution at $(0, x) \forall x \in \mathbb{R}^{n}$, and either $v_{2}(T, x) \geq v_{1}(T, x)$ or $v_{2}$ is a supersolution at $(T, x) \forall x \in \mathbb{R}^{n}$. By (i) in Proposition 2.1 one has

$$
\frac{w_{0}^{\beta}}{1+t}+\bar{\ell}\left(t, x, w_{0}, w\right) \mu \geq \frac{w_{0}^{\beta}}{1+t}+\mu \ell_{0}|w|^{\beta}-\mu \ell_{1} w_{0}^{\beta},
$$


for all $\left(t, x, w_{0}, w\right)$ which, by the choice of $\mu$, implies that there exists $\eta>0$ such that

$$
\frac{w_{0}^{\beta}}{1+t}+\bar{\ell}\left(t, x, w_{0}, w\right) \mu \geq \eta, \quad \forall\left(w_{0}, w\right) \in S_{+}^{m} .
$$

Therefore, for every $(t, x) \in[0, T] \times \mathbb{R}^{m}$, the number

$$
k(t, x) \doteq \min _{\left(w_{0}, w\right) \in S_{+}^{m}}\left(\frac{w_{0}^{\beta}}{1+t}+\bar{\ell}\left(t, x, w_{0}, w\right) \mu\right)
$$

is positive, so equation (2.6) is equivalent to

$$
\begin{array}{r}
v+\max _{\left(w_{0}, w\right) \in\left(\left[0,+\infty[\times C) \cap S_{+}^{m}\right.\right.} \frac{1}{k}\left(v\left(\frac{w_{0}^{\beta}}{1+t}+\bar{\ell} \mu-k\right)-\nabla_{t} v w_{0}^{\beta}\right. \\
\left.-\left\langle\bar{f}, \nabla_{x} v\right\rangle-\bar{\ell}(1+t) \mu\right)=0 .
\end{array}
$$

Equation (2.7) verifies the conditions assumed in Theorem 1.1 in [4]. By applying the latter to the functions $v_{1}$ and $v_{2}$, we obtain

$$
v_{1}(t, x) \leq v_{2}(t, x), \quad \forall(t, x) \in[0, T] \times \mathbb{R}^{n},
$$

which, in view of the monotonicity of $\Lambda$, yields the theorem.

Corollary 2.2 (Uniqueness and continuity of the solution of $\left(\mathrm{HJ}_{e}\right)$ ). Assume $\left(\mathrm{A}_{1}\right)-\left(\mathrm{A}_{5}\right)$. Then, if $\alpha<\beta[$ resp. $\alpha=\beta]$, problem $\left(\mathrm{HJ}_{e}\right)-\left(\mathrm{BC}_{e}\right)\left[\right.$ resp. $\left(\mathrm{HJ}_{e}\right)-$ $\left.\left(\mathrm{BC}_{e_{m}}\right)\right]$ admits at most one bounded below, viscosity solution. Moreover, if a solution exists, this solution is continuous.

Proof. The corollary follows from Theorem 2.4 and Lemma 2.1 below.

The following result establishes a relation between subsolutions and supersolutions of $(\mathrm{HJ})$ and $\left(\mathrm{HJ}_{e}\right)$.

Theorem 2.5. Assume $\left(\mathrm{A}_{1}\right)-\left(\mathrm{A}_{4}\right)$, with $\alpha \leq \beta$.

(i) Let $u:[0, T] \times \mathbb{R}^{n} \rightarrow \mathbb{R}$ be upper semicontinuous and let $(t, x) \in$ $[0, T] \times \mathbb{R}^{n}$. Then $u$ is a viscosity subsolution of $\left(\mathrm{HJ}_{e}\right)$ at $(t, x)$ if and only if it is a viscosity subsolution of $(\mathrm{HJ})$ at $(t, x)$.

(ii) Let $u:[0, T] \times \mathbb{R}^{n} \rightarrow \mathbb{R}$ be lower semicontinuous and let $(t, x) \in[0, T] \times$ $\mathbb{R}^{n}$. Then $u$ is a viscosity supersolution of $\left(\mathrm{HJ}_{e}\right)$ at $(t, x)$ if and only if it is a viscosity supersolution of $(\mathrm{HJ})$ at $(t, x)$. 
Remark 2.6. It is remarkable that even for $\alpha=\beta$ the supersolution condition for $\left(\mathrm{HJ}_{e}\right)$ implies an analogous condition for $(\mathrm{HJ})$. This is strictly connected with the special form of the Hamiltonian $H_{e}$. In fact, for a less specific regularized Hamiltonian this implication is, in general, false (see Remark II.2 in [6]).

Proof of Theorem 2.5. Let us begin by proving (i). Assume that $u$ is a subsolution of $(\mathrm{HJ})$ at $(t, x)$. Then, if $\varphi \in C^{\infty}\left(\mathbb{R}^{1+n}\right)$ is such that $u-\varphi$ has a local maximum at $(t, x)$, one has

$$
H_{*}(t, x, \nabla \varphi(t, x)) \leq 0 .
$$

This implies that there exists a sequence $\left(s_{n}, y_{n}, p_{0 n}, p_{n}\right)$ approaching $(t, x, \nabla \varphi(t, x))$ such that

$$
\left(-p_{0 n}-\left\langle p_{n}, f\left(s_{n}, y_{n}, c\right)\right\rangle-\ell\left(s_{n}, y_{n}, c\right)\right) \leq \frac{1}{n},
$$

for every $c \in C$ and every $n \in \mathbb{N}$. Let us write $w / w_{0}$ in place of $c$, with $\left(w_{0}, w\right) \in$ $\left(\left[0,+\infty[\times C) \cap S_{+}^{m}\right.\right.$. Multiplying the inequality above by $w_{0}^{\beta}$, we get

$$
\left(-p_{0 n} w_{0}^{\beta}-\left\langle p_{n}, \bar{f}\left(s_{n}, y_{n}, w_{0}, w\right)\right\rangle-\bar{\ell}\left(s_{n}, y_{n}, w_{0}, w\right)\right) \leq \frac{1}{n} w_{0}^{\beta} \leq \frac{1}{n} .
$$

Since $\left(w_{0}, w\right)$ is arbitrary in the interior of $\left(\left[0,+\infty[\times C) \cap S_{+}^{m}\right.\right.$, by the continuity of $H_{e}$ we get

$$
H_{e}(t, x, \nabla \varphi(t, x)) \leq 0 .
$$

Therefore $u$ is a subsolution of $\left(\mathrm{HJ}_{e}\right)$ at $(t, x)$. By simply reversing the above arguments, we obtain the converse implication.

Let us prove (ii). The proof that every supersolution of $(\mathrm{HJ})$ at $(t, x)$ is a supersolution of $\left(\mathrm{HJ}_{e}\right)$ at $(t, x)$ is quite similar to the proof of the analogous implication in the case of subsolutions. For this reason we omit it.

The proof that every supersolution of $\left(\mathrm{HJ}_{e}\right)$ at $(t, x)$ is in fact a supersolution of $(\mathrm{HJ})$ is less straightforward. Let $u$ be a supersolution of $\left(\mathrm{HJ}_{e}\right)$ at $(t, x)$ and let $\varphi \in C^{\infty}$ be such that $u-\varphi$ has a local minimum at $(t, x)$. Let $\left(\bar{w}_{0}, \bar{w}\right)$ be a pair such that

$$
\begin{aligned}
& \left(-\nabla_{t} \varphi(t, x) \bar{w}_{0}^{\beta}-\left\langle\nabla_{x} \varphi(t, x), \bar{f}\left(t, x, \bar{w}_{0}, \bar{w}\right)\right\rangle-\bar{\ell}\left(t, x, \bar{w}_{0}, \bar{w}\right)\right) \\
= & \sup _{\left(\left[0,+\infty[\times C) \cap S_{+}^{m}\right.\right.}\left\{-\nabla_{t} \varphi(t, x) w_{0}^{\beta}-\left\langle\nabla_{x} \varphi(t, x), \bar{f}\left(t, x, w_{0}, w\right)\right\rangle-\bar{\ell}\left(t, x, w_{0}, w\right)\right\} \\
\geq & 0 .
\end{aligned}
$$


Let $\left(h_{n}\right)_{n \in \mathbb{N}},\left(k_{n}\right)_{n \in \mathbb{N}}$ be positive sequences approaching zero, and let us define

$$
p_{0 n} \doteq \nabla_{t} \varphi(t, x)-h_{n} \quad \text { and } \quad p_{n} \doteq \nabla_{x} \varphi(t, x)-k_{n} \bar{f}\left(t, x, \bar{w}_{0}, \bar{w}\right) .
$$

Observe that for every $n \in \mathbb{N}, h_{n} \bar{w}_{0}^{\beta}+k_{n}\left|\bar{f}\left(t, x, \bar{w}_{0}, \bar{w}\right)\right|^{2}>0$, in that it cannot happen that both $\bar{w}_{0}$ and $\left|\bar{f}\left(t, x, \bar{w}_{0}, \bar{w}\right)\right|$ are equal to zero. Indeed, if by contradiction $\bar{w}_{0}=\left|f\left(t, x, \bar{w}_{0}, \bar{w}\right)\right|=0$, then $|\bar{w}|>0$. By $\left(\mathrm{A}_{e_{4}}\right)$ (see Proposition 2.1) this yields $\bar{\ell}\left(t, x, \bar{w}_{0}, \bar{w}\right)>0$, which contradicts (2.8). Therefore there exists a positive constant $m_{n}$ such that

$$
\sup _{\left(w_{0}, w\right) \in\left(\left[0,+\infty[\times C) \cap S_{+}^{m}\right.\right.}\left\{-p_{0 n} w_{0}^{\beta}-\left\langle p_{n}, \bar{f}\left(t, x, w_{0}, w\right)\right\rangle-\bar{\ell}\left(t, x, w_{0}, w\right)\right\}=m_{n} .
$$

In particular there exists a sequence $\left(w_{0 n}, w_{n}\right)$ verifying $w_{0 n}>0$ and such that

$$
\left(-p_{0 n}-\left\langle p_{n}, f\left(t, x, c_{n}\right)\right\rangle-\ell\left(t, x, c_{n}\right)\right)>\frac{m_{n}}{2 w_{0 n}{ }^{\beta}}>0,
$$

where we have set $c_{n} \doteq w_{n} / w_{0 n}$. Hence

$$
\sup _{c \in C}\left\{-p_{0 n}-\left\langle p_{n}, f(t, x, c)\right\rangle-\ell(t, x, c)\right\}>0
$$

which implies

$$
H^{*}(t, x, \nabla \varphi(t, x)) \geq 0 \text {. }
$$

Hence $u$ is a supersolution of $(\mathrm{HJ})$, and the proof is concluded.

Lemma 2.1. Assume hypotheses $\left(\mathrm{A}_{1}\right)-\left(\mathrm{A}_{5}\right)$. Let $u$ be a viscosity supersolution of $\left(\mathrm{HJ}_{e}\right)$ in $] 0, T\left[\times \mathbb{R}^{n}\right.$, continuous on $\left(\{0\} \times \mathbb{R}^{n}\right)$. Then $u$ is a viscosity supersolution at $(0, x)$, for every $x \in \mathbb{R}^{n}$.

Proof. The proof of this lemma is based on standard arguments (see e.g. [14]) so we omit it.

\section{Unbounded CONTROL SYSTEMS AND $\beta$-POWER REPARAMETERIZATIONS}

For every $\bar{t} \in[0, T]$, let $C(\bar{t})$ denote the set of Borel-measurable maps which belong to $L^{\beta}\left([\bar{t}, T], \mathbb{R}^{m}\right)$ and take values in $C$. For every $(\bar{t}, \bar{x}) \in[0, T] \times \mathbb{R}^{n}$ and every $c \in C(\bar{t})$, let us denote the unique solution of the Cauchy problem

$$
\left\{\begin{array}{l}
\dot{x}=f(t, x, c) \quad \text { for } t \in[\bar{t}, T] \\
x(\bar{t})=\bar{x},
\end{array}\right.
$$


(where the dot means differentiation with respect to $t$ ) by $x_{(\bar{t}, \bar{x})}[c](\cdot)$ (or, if the initial data are known by the contest, by $x[c](\cdot))$. For every $(\bar{t}, \bar{x}, c) \in[0, T] \times$ $\mathbb{R}^{n} \times C(\bar{t})$ let us consider the cost functional

$$
J(\bar{t}, \bar{x}, c) \doteq \int_{\bar{t}}^{T} \ell(t, x[c](t), c(t)) d t+g(x[c](T))
$$

and let us define the value function as the map

$$
V:\left[0, T\left[\times \mathbb{R}^{n} \rightarrow \mathbb{R},\right.\right.
$$

which associates the infimum value of $J$ to each initial condition $(\bar{t}, \bar{x})$ :

$$
V(\bar{t}, \bar{x}) \doteq \inf _{c \in C(\bar{t})} J(\bar{t}, \bar{x}, c)
$$

Theorem 3.1. Assume $\left(\mathrm{A}_{1}\right)-\left(\mathrm{A}_{5}\right)$. Then the following statements hold true:

(i) $V$ is continuous and can be continuously extended to $[0, T] \times \mathbb{R}^{n}$. Actually, if $\alpha<\beta$,

$$
\lim _{t \rightarrow T} V(t, x)=g(x) \quad \text { for all } x \in \mathbb{R}^{n},
$$

so (the extension to $t=T$ of $) V$ verifies $(\mathrm{BC})$.

(ii) $V$ is solution of $(\mathrm{HJ})$ in $] 0, T\left[\times \mathbb{R}^{n}\right.$.

(iii) For $\alpha=\beta$ the extension of $V$ to $t=T$, verifies $\left(\mathrm{BC}_{m}\right)$.

When a suitable condition on the data is in force we can prove that $V$ is a solution of the Cauchy problem (HJ)-(BC) (and not only of $(\mathrm{HJ})-\left(\mathrm{BC}_{m}\right)$ ) even in the case when $\alpha=\beta$. More precisely, for $\alpha=\beta$, let us assume that

$(\mathrm{NFJ})$

$$
\int_{0}^{1} \ell^{\infty}(T, y(s), w(s)) d s \geq g(x)-g(y(1))
$$

for all $x \in \mathbb{R}^{n}$ and all $L^{\infty}$ controls $w:[0,1] \rightarrow C$, where $y(\cdot)$ denotes the solution to the Cauchy problem

$$
\left\{\begin{array}{l}
\frac{d y}{d s}(s)=f^{\infty}(T, y(s), w(s)), \quad \forall s \in[0,1], \\
y(0)=x
\end{array} .\right.
$$

and $\ell^{\infty}$ and $f^{\infty}$ are the recession functions defined in the Introduction. Let us call assumption (NFJ) the no-final-jump condition. For instance, one can easily check 
that (NFJ) is satisfied when $g$ is Lipschitz continuous with Lipschitz constant $L_{\mathscr{g}}$, $\left(\mathrm{A}_{3}\right)$ is verified with $M_{1}=0$, and

$$
\ell_{0} \geq M_{2} L_{g}
$$

where $M_{2}$ and $\ell_{0}$ are the constants appearing in $\left(\mathrm{A}_{3}\right)$ and $\left(\mathrm{A}_{4}\right)$, respectively.

Theorem 3.2. For $\alpha=\beta$, let us assume condition (NFJ). Then (the continuous extension to $t=T$ of $) V$ is a solution of the Cauchy problem (HJ)-(BC).

In view of Corollary 2.1, one obtains the following Corollary:

Corollary 3.1. Assume $\left(\mathrm{A}_{1}\right)-\left(\mathrm{A}_{5}\right)$. If $\alpha<\beta$ [resp. $\left.\alpha=\beta\right]$ then $V$ is the unique solution of $(\mathrm{HJ})-(\mathrm{BC})\left[\right.$ resp. $\left.(\mathrm{HJ})-\left(\mathrm{BC}_{m}\right)\right]$. Moreover if $\alpha=\beta$ and the no-finaljump condition (NFJ) is in force, $V$ is also the unique solution of $(\mathrm{HJ})-(\mathrm{BC})$.

In order to prove Theorem 3.1, let us introduce an auxiliary optimal control problem which is obtained from the original one via a $\beta$-power reparameterization of time.

For every $\bar{t} \in[0, T]$ let us introduce the following sets of space-time controls

$$
\Gamma(\bar{t}) \doteq\left\{\left(w_{0}, w\right) \in \mathcal{B}([0,1],[0,+\infty) \times C) \text { such that } \bar{t}+\int_{0}^{1} w_{0}^{\beta}(s) d s=T\right\}
$$

and

$$
\Gamma^{+}(\bar{t}) \doteq\left\{\left(w_{0}, w\right) \in \Gamma(\bar{t}) \quad \text { such that } \quad w_{0}>0 \text { a.e. }\right\}
$$

where $\mathcal{B}([0,1],[0,+\infty) \times C)$ is the set of $L^{\infty}$, Borel maps, which take values in $\left[0,+\infty[\times C\right.$. If $\alpha<\beta$ [resp. $\alpha=\beta]$, for every $(\bar{t}, \bar{x}) \in[0, T] \times \mathbb{R}^{n}$ and every $\left(w_{0}, w\right) \in \Gamma^{+}(\bar{t})\left[\operatorname{resp} .\left(w_{0}, w\right) \in \Gamma(\bar{t})\right]$, let us denote by $(t, y)_{(\bar{t}, \bar{x})}\left[w_{0}, w\right](\cdot)$ the solution of the Cauchy problem

$\left(\mathrm{E}_{e}\right)$

$$
\left\{\begin{array}{l}
t^{\prime}(s)=w_{0}^{\beta}(s) \\
y^{\prime}(s)=\bar{f}\left(t(s), y(s), w_{0}(s), w(s)\right) \\
(t(0), y(0))=(\bar{t}, \bar{x}),
\end{array}\right.
$$

where the parameter $s$ belongs to the interval $[0,1]$ and the prime denotes differentiation with respect to $s$. When the initial conditions are meant by the context, we shall write $(t, y)\left[w_{0}, w\right](\cdot)$ instead of $(t, y)_{(\bar{t}, \bar{x})}\left[w_{0}, w\right](\cdot)$. Let us consider 
the following cost functional

$$
J_{e}\left(\bar{t}, \bar{x}, w_{0}, w\right) \doteq \int_{0}^{1} \bar{\ell}\left((t, y)\left[w_{0}, w\right], w_{0}, w\right)(s) d s+g\left(y\left[w_{0}, w\right](1)\right)
$$

and the corresponding value function

$$
\begin{gathered}
V_{e}:[0, T] \times \mathbb{R}^{n} \rightarrow \mathbb{R} \\
V_{e}(\bar{t}, \bar{x}) \stackrel{\doteq}{\inf _{\left(w_{0}, w\right) \in \Gamma(\bar{t})}} J_{e}\left(\bar{t}, \bar{x}, w_{0}, w\right) .
\end{gathered}
$$

In view of Theorem 3.3 below, the optimal control problem introduced above is in fact an extension of the original one.

In order to establish a connection between the original system (E) and the extended system $\left(\mathrm{E}_{e}\right)$, for every $c \in C(\bar{t})$ let us introduce the following $\beta$-power reparameterization of time. For every $t \in[\bar{t}, T]$ let us set

$$
s(t) \doteq \frac{\int_{\bar{t}}^{t}\left(1+|c(\tau)|^{\beta}\right) d \tau}{\int_{\bar{t}}^{T}\left(1+|c(\tau)|^{\beta}\right) d \tau}
$$

and let

$$
t:[0,1] \rightarrow[\bar{t}, T]
$$

denote the inverse of $s(\cdot)$. Proposition 3.1 below establishes a substantial equivalence between the original system $(\mathrm{E})$ and the extended system $\left(\mathrm{E}_{e}\right)$ when spacetime controls belonging to the subset $\Gamma^{+}(\bar{t})$ are implemented.

Proposition 3.1 (Embedding). Let $(\bar{t}, \bar{x}) \in\left[0, T\left[\times \mathbb{R}^{n}, c \in C(\bar{t})\right.\right.$ and let $x(\cdot)$ be the corresponding solution of $(\mathrm{E})$. Let $t(\cdot)$ be the map defined as above. Then the map $(t, y)(s) \doteq(t(s), x \circ t(s))$ is the (unique) solution of $\left(\mathrm{E}_{e}\right)$ corresponding to the control:

$$
\left(w_{0}, w\right)(s) \doteq \sqrt[\beta]{t^{\prime}(s)} \cdot(1, c \circ t(s))\left(\in \Gamma^{+}(\bar{t})\right)
$$

On the other hand, let $\left(w_{0}, w\right)$ be a space-time control belonging to $\Gamma^{+}(\bar{t})$ and set $(t, y)(s) \doteq(t, y)_{(\bar{t}, \bar{x})}\left[w_{0}, w\right](s)$. Then the relation

$$
c(t) \doteq \frac{w}{w_{0}} \circ s(t)
$$


where $s(t)$ is the inverse of the function $t(s)$, defines almost everywhere in $[\bar{t}, T]$ a single valued map $c$ which can be extended to a Borel measurable map in $[\bar{t}, T]$. Moreover, setting $x(\cdot) \doteq x_{(\bar{t}, \bar{x})}[c](\cdot)$, one has

$$
x(t)=y \circ s(t)
$$

for every $t \in[\bar{t}, T]$ and

$$
J(\bar{t}, \bar{x}, c)=J_{e}\left(\bar{t}, \bar{x}, w_{0}, w\right) .
$$

Proof. Let us observe that the control $\left(w_{0}, w\right)$ in the first part of the thesis actually belongs to $\Gamma^{+}(\bar{t})$. Indeed one has

$$
\int_{0}^{1} w_{0}^{\beta}(s) d s=\int_{0}^{1} t^{\prime}(s) d s=T-\bar{t}
$$

and

$\left|\left(w_{0}, w\right)\right|(s)=\sqrt[\beta]{\frac{\int_{\bar{t}}^{T}\left(1+|c(\tau)|^{\beta}\right) d \tau}{1+|c \circ t(s)|^{\beta}}}|(1, \cot (s))| \leq \sqrt{2} \sqrt[\beta]{\int_{\bar{t}}^{T}(1+|c(\tau)| \beta) d \tau}$.

Then the result easily follows from the fact that $\left(\mathrm{E}_{e}\right)$ has a unique solution for every given control $\left(w_{0}, w\right) \in \Gamma(\bar{t})$.

The fact that $c(\cdot)$ is defined almost everywhere follows from the absolute continuity of $t(\cdot)$. Moreover, since $w(\cdot), w_{0}(\cdot)$ and $s(\cdot)$ are Borel-measurable maps it follows that $c(\cdot)$ is a Borel-measurable map as well. (More precisely $c(\cdot)$ can be extended to a Borel-measurable map, e.g. by setting $c(t) \equiv c \circ s(1)$ when $w \circ s(t)=0)$.

Proposition 3.2 below shows that the set of trajectories graphs of (E) is dense in the set of trajectories of $\left(\mathrm{E}_{e}\right)$.

Proposition 3.2 (Density). Let $\left(w_{0}, w\right) \in \Gamma(\bar{t})$. Then there exists a sequence $\left(w_{0 n}, w\right) \in \Gamma^{+}(\bar{t})$ such that setting $\left(t_{n}, y_{n}\right)(s) \doteq(t, y)\left[w_{0 n}, w\right](s)$ one has

(i) $y_{n} \circ t_{n}^{-1}(t)=x\left[c_{n}\right](t)$, for every $t \in[\bar{t}, T]$, where $c_{n} \doteq\left(w / w_{0_{n}}\right) \circ$ $t_{n}^{-1}$

(ii) the sequence $\left(t_{n}, y_{n}\right)$ converges uniformly to $(t, y)\left[w_{0}, w\right]$;

(iii) the sequence $J\left(\bar{t}, \bar{x}, c_{n}\right)$ converges to $J_{e}\left(\bar{t}, \bar{x}, w_{0}, w\right)$. 
Proof. Let $\left(w_{0}, w\right) \in \Gamma(t)$. For every $n \in \mathbb{N}$ and $s \in[0,1]$ let us define:

$$
w_{0 n} \doteq \sqrt[\beta]{\frac{T-\bar{t}}{T-\bar{t}+\frac{1}{n}}\left(w_{0}^{\beta}+\frac{1}{n}\right)}
$$

and let us observe that $\left(w_{0 n}, w\right) \in \Gamma^{+}(\bar{t})$. Hence (i) follows from Proposition 3.1. Let us prove that the sequence $\left(t_{n}, y_{n}\right)=(t, y)\left[w_{0_{n}}, w\right]$ converges uniformly to $(t, y)\left[w_{0}, w\right]$. Let us observe that

$$
\left|w_{0_{n}}^{\beta}(s)-w_{0}^{\beta}(s)\right| \leq \frac{1}{n} \frac{T-\bar{t}+\left\|w_{0}^{\beta}\right\|_{\infty}}{T-\bar{t}+\frac{1}{n}} \quad \forall s \in[0,1] .
$$

This and Gronwall's lemma imply that there exists a compact $Q \subset[0, T] \times \mathbb{R}^{n}$ containing the trajectories $\left(t_{n}, y_{n}\right)$ and $(t, y)$. Then one concludes via standard arguments, essentially Gronwall's Lemma and the Dominated Convergence Theorem. Finally (iii) is an obvious consequence of the previous steps.

\section{Theorem 3.3.}

(i) For every $(t, x) \in\left[0, T\left[\times \mathbb{R}^{n}\right.\right.$ one has $V_{e}(t, x)=V(t, x)$;

(ii) the map $V_{e}:[0, T] \times \mathbb{R}^{n} \rightarrow \mathbb{R}$ is continuous.

Proof. The proof of (i) is a straightforward consequence of Proposition 3.2. Moreover, in view of Lemma 3.1 below, the map $V_{e}$ is continuous on $\{T\} \times \mathbb{R}^{n}$. Finally, by (i) and Theorem 4.1 below, $V_{e}$ is continuous on $\left[0, T\left[\times \mathbb{R}^{n}\right.\right.$ as well.

Lemma 3.1. Assume hypotheses $\left(\mathrm{A}_{1}\right)-\left(\mathrm{A}_{5}\right)$. Then the function $V_{e}$ is continuous on $\{T\} \times \mathbb{R}^{n}$.

Proof. The continuity of $V_{e}$ in the variable $x$ is proved in the first part of the proof of Theorem 4.1 (see (4.5)). As for the continuity in $t$, in (4.6) below the quantity $V_{e}(t, x)-V_{e}(T, x)$ is proved to be majorized by a nonnegative map which tends to zero with $T-t$. Hence it remains only to estimate the difference $V_{e}(T, x)-V_{e}(t, x), t \leq T$.

Let us fix $\varepsilon>0$ and let us consider a space-time control $\left(\widetilde{w}_{0}, \widetilde{w}\right) \in \Gamma(t)$, such that, setting $(\tilde{t}, \tilde{x}) \doteq(t, y)_{(t, x)}\left[\widetilde{w}_{0}, \widetilde{w}\right]$, one has

$$
V_{e}(t, x) \geq \int_{0}^{1} \bar{\ell}\left(\tilde{t}(s), \tilde{x}(s), \widetilde{w}_{0}(s), \widetilde{w}(s)\right) d s+g(\tilde{x}(1))-\varepsilon .
$$


Setting $(\stackrel{\vee}{t}, \check{x}) \doteq(t, y)_{(T, x)}[0, \widetilde{w}]$, one obtains

$$
\begin{aligned}
V_{e}(T, x)-V_{e}(t, x) \leq \int_{0}^{1} \bar{\ell}(T, \grave{x}, 0, \widetilde{w})(s) d s+g(\check{x}(1)) \\
\quad-\int_{0}^{1} \bar{\ell}\left(\tilde{t}, \tilde{x}, \widetilde{w}_{0}, \widetilde{w}\right)(s) d s-g(\tilde{x}(1))+\varepsilon .
\end{aligned}
$$

As observed at the beginning of the proof of Theorem 4.1 below, whenever $x$ belongs to a compact subset $B \subset \mathbb{R}^{n}$, we can restrict the set of space-time controls to those satisfying

$$
\int_{0}^{1}\left(w_{0}(s)+|w(s)|\right)^{\beta} d s \leq K,
$$

where $K$ is a suitable constant depending on $B$. Actually, in view of the parameterfree character of system $\left(\mathrm{E}_{e}\right)$, the set of controls can be restricted to those satisfying $\left|\left(w_{0}, w\right)(s)\right| \leq K_{1} \forall s \in[0,1]$, where $K_{1}$ still depends on $B$ (see e.g. [20] for the case $\alpha=\beta=1)$. Therefore by Gronwall's Lemma, we can assume that there is a ball $B[0, R]$ containing all the trajectories issuing from $x \in B$. One has

$$
\begin{aligned}
|\tilde{x}(s)-\grave{x}(s)| \leq \int_{0}^{1}\left|\bar{f}\left(\tilde{t}, \tilde{x}, \widetilde{w}_{0}, \widetilde{w}\right)(s)-\bar{f}(T, \grave{x}, 0, \widetilde{w})(s)\right| d s \\
\leq \int_{0}^{1}\left|\bar{f}\left(\tilde{t}, \tilde{x}, \widetilde{w}_{0}, \widetilde{w}\right)(s)-\bar{f}\left(T, \tilde{x}, \widetilde{w}_{0}, \widetilde{w}\right)(s)\right| d s \\
\quad+\int_{0}^{1}\left|\bar{f}\left(T, \tilde{x}, \widetilde{w}_{0}, \widetilde{w}\right)(s)-\bar{f}\left(T, \grave{x}, \widetilde{w}_{0}, \widetilde{w}\right)(s)\right| d s \\
\quad+\int_{0}^{1}\left|\bar{f}\left(T, \grave{x}, \widetilde{w}_{0}, \widetilde{w}\right)(s)-\bar{f}(T, \grave{x}, 0, \widetilde{w})(s)\right| d s .
\end{aligned}
$$

Observe that the last integral can be majorized by a term of the form $\omega(T-t)$, where $\omega$ is a modulus (see for instance [21] Lemma 3.1). By (4.4) below we obtain

$$
\begin{array}{r}
|\tilde{x}(s)-\check{x}(s)| \leq(T+1)(K+1)\left[\omega_{f}(|T-t|)+\int_{0}^{1} L_{R}|\tilde{x}(s)-\check{x}(s)| d s\right] \\
+\omega(|T-t|),
\end{array}
$$

which yields

$$
|\tilde{x}(s)-\stackrel{v}{x}(s)| \leq\left[(T+1)(K+1)\left(\omega_{f}(|T-t|)+\omega(|T-t|)\right] e^{L_{R}(T+1)(K+1)},\right.
$$

where $L_{R}$ is the determination of $L$ in $\left(\mathrm{A}_{e_{1}}\right)$ when $Q=[0, T] \times B[0, R]$. The 
second integral in

$$
\begin{aligned}
V_{e}(T, x) & -V_{e}(t, x) \leq K \int_{0}^{1} \omega_{\ell}(|(\tilde{t}(s), \tilde{x}(s))-(T, \grave{x}(s))|) d s \\
& +\int_{0}^{1}\left|\bar{\ell}(T, \grave{x}, 0, \widetilde{w})(s)-\bar{\ell}\left(T, \grave{x}, \widetilde{w_{0}}, \widetilde{w}\right)(s)\right| d s+\omega_{g}(|\tilde{x}(1)-\grave{x}(1)|)+\varepsilon
\end{aligned}
$$

approaches zero as $t$ approaches $T$ (see [21] Lemma 3.1). This fact, together with the estimate obtained for $|\tilde{x}(s)-\ddot{x}(s)|$ provides the required estimate for $V_{e}(T, x)-V_{e}(t, x)$.

Proof of Theorem 3.1. The first statement in (i) follows from Theorem 3.3. Moreover, if $\alpha<\beta$, for every $x \in \mathbb{R}^{n}$ and every $\left(w_{0}, w\right) \in \Gamma(T)$ one has

$$
J_{e}\left(T, x, w_{0}, w\right) \geq \int_{0}^{1} \ell_{0}|w(s)|^{\beta} d s+g(x)
$$

which implies $V_{e}(T, x)=g(x)$. This fact and Theorem 3.3 imply the second statement in (i).

Let us prove (ii). With standard arguments one can show that $V_{e}$ is a viscosity solution of $\left(\mathrm{HJ}_{e}\right)$ on the open subset $] 0, T\left[\times \mathbb{R}^{n}\right.$. Moreover, by Theorem 3.3, $V_{e}=V$ on $\left[0, T\left[\times \mathbb{R}^{n}\right.\right.$. Hence, in view of Theorem $2.5, V$ is a viscosity solution of $(\mathrm{HJ})$ in $] 0, T\left[\times \mathbb{R}^{n}\right.$.

To prove (iii) we argue as in [22]. More precisely, relying on the special Dynamic Programming Principle provided by Proposition 3.4 below, one can easily prove that the value function of the extended problem verifies $\left(\mathrm{BC}_{m}\right)$. Then, in view of Theorem 2.5, the extension of $V\left(=V_{e}\right)$ verifies $\left(\mathrm{BC}_{m}\right)$ as well.

Proof of Theorem 3.2. Let us observe that the no-final-jump condition (NFJ) implies that the implementation of impulsive controls $(0, w), w \neq 0$, does not yield any advantage with respect to the control $(0,0)$. Indeed condition (NFJ) implies that for every $x \in \mathbb{R}^{n}$,

$$
\inf _{(0, w) \in \Gamma(T)} \int_{0}^{1} \bar{\ell}(T, y, 0, w)(s) d s+g(x(1))-g(x) \geq 0,
$$

while $\int_{0}^{1} \bar{\ell}(T, y, 0,0)=0$ and $y[0,0](s)=x, \forall s \in[0,1]$

Proposition 3.4 (Dynamic Programming Principle for $V_{e}$ at $t=T$ ). Let $\alpha=$ $\beta$. Let $x \in \mathbb{R}^{n}$ and $\eta>0$ be such that $g(x)-V_{e}(T, x)=\eta$. Then there exists 
$\sigma>0$ such that, for all $s \in[0,1]$, we have

$$
\begin{aligned}
V_{e}(T, x)=\inf _{\left(w_{0}, w\right) \in \Gamma_{\sigma}(T)}\left\{\int _ { 0 } ^ { s } \overline { \ell } \left((t, x)\left[w_{0}, w\right],\right.\right. & \left.w_{0}, w\right)\left(s^{\prime}\right) d s^{\prime} \\
& \left.+V_{e}\left((t, x)\left[w_{0}, w\right](s)\right)\right\}
\end{aligned}
$$

where $\Gamma_{\sigma}\left(t_{0}\right) \doteq\left\{\left(w_{0}, w\right) \in \Gamma\left(t_{0}\right): \int_{0}^{1}\left(w_{0}^{\beta}(s)+|w(s)|^{\beta}\right) d s \geq \sigma\right\}$

Proof. The theorem follows straightforwardly by the fact that there exists $\sigma$ such that

(3.2) $V_{e}(T, x)$

$$
=\inf _{\left(w_{0}, w\right) \in \Gamma_{\sigma}(T)} \int_{0}^{1} \bar{\ell}\left((t, x)\left[w_{0}, w\right], w_{0}, w\right)(s) d s+g\left(x\left[w_{0}, w\right](1)\right) .
$$

Let us prove (3.2). It is clear that $V_{e}(T, x)$ is less than or equal to the right-hand side of (3.2), for $\Gamma_{\sigma}(T) \subset \Gamma(T)$. To prove the converse inequality, assume by contradiction that for every minimizing sequence of controls $\left(w_{0 n}, w_{n}\right) \in \Gamma(T)$ verifying

$$
V_{e}(T, x)+\frac{1}{n} \geq \int_{0}^{1} \bar{\ell}\left((t, x)\left[w_{0 n}, w_{n}\right], w_{0 n}, w_{n}\right)(s) d s+g\left(x\left[w_{0 n}, w_{n}\right](1)\right)
$$

and for every $\sigma>0$ there exists $\bar{n}$ such that $\forall n \geq \bar{n}$ one has $\int_{0}^{1}\left|w_{n}(s)\right|^{\beta} d s<\sigma$ (notice that $w_{0_{n}}(s)=0 \quad \forall s \in[0,1]$ and $\forall n \in \mathbb{N}$ ). Then there exists a constant $\bar{K}$ such that

$$
\left|\left(x\left[w_{0 n}, w_{n}\right](s)-x\right)\right| \leq \bar{K} \sigma .
$$

Moreover, by the continuity and the $\beta$-homogeneity of $\bar{\ell}$, there is a constant $\tilde{K}$ such that

$$
\int_{0}^{1} \bar{\ell}\left((t, x)\left[w_{0 n}, w_{n}\right], w_{0 n}, w_{n}\right)(s) d s \leq \tilde{K} \sigma .
$$

Let us choose $\sigma$ so that

$$
\left|g\left(x\left[w_{0 n}, w_{n}\right](1)\right)-g(x)\right| \leq \omega_{g}(\bar{K} \sigma) \leq \frac{\eta}{4} \quad \text { and } \quad \tilde{K} \sigma \leq \frac{\eta}{4} .
$$


Then, by choosing $n \geq \max \{\bar{n}, 4 / \eta\}$, we have that

$$
\begin{aligned}
\eta=g(x)-V_{e}(T, x) \leq-\int_{0}^{1} \bar{\ell}( & \left.(t, x)\left[w_{0 n}, w_{n}\right], w_{0 n}, w_{n}\right)(s) d s \\
& \quad-g\left(x\left[w_{0 n}, w_{n}\right](1)\right)+g(x)+\frac{\eta}{4} \leq \frac{3 \eta}{4},
\end{aligned}
$$

which is a contradiction, for $\eta>0$.

\section{REGULARITY OF THE SOLUTION}

We have proved that, when $\alpha<\beta$ [resp. $\alpha=\beta$ ], the value function $V$ introduced in the previous section is the unique solution of the Cauchy problem $(\mathrm{HJ})-(\mathrm{BC})$ [resp. $\left.(\mathrm{HJ})-\left(\mathrm{BC}_{m}\right)\right]$. Now we exploit this characterization to prove some regularity properties of this solution. Regularity properties of the solution were studied e.g. by P. L. Lions [18] and G. Barles [5] [7], in the case of Hamiltonians that, in particular, are continuous and superlinear in the adjoint variable $p$, uniformly in $x$. Moreover, under a set of hypotheses on $f$ and $\ell$, which in particular imply (1.2), sharp regularity estimates are given by M. Bardi and F. Da Lio in [3]. For a different class of problems, still verifying hypothesis (1.2), regularity results are provided in [1].

The main point of the results below consists in the fact that they hold also when $\mathcal{H}$ is allowed to depend on $p$ non uniformly with respect to $x$. In particular, condition (1.2) is not assumed. Moreover, regularity results are proved even if $\alpha=\beta$, a case in which the Hamiltonian is, in general, discontinuous (see also [21], [24] and [6]).

Theorem 4.1. Assume $\left(\mathrm{A}_{1}\right)-\left(\mathrm{A}_{5}\right)$ and fix $R>0$. Then there exists $R^{\prime} \geq R$ and positive constants $B_{1}, B_{2}$ such that

$$
\left|V\left(t, x_{1}\right)-V\left(t, x_{2}\right)\right| \leq B_{1} \omega_{\ell}\left(B_{2}\left|x_{2}-x_{1}\right|\right)+\omega_{g}\left(B_{2}\left|x_{2}-x_{1}\right|\right)
$$

for every $\left(t, x_{1}\right)\left(t, x_{2}\right) \in\left[0, T\left[\times B[0 ; R]\right.\right.$, where $\omega_{\ell}$ and $\omega_{g}$ are the modulus appearing in $\left(\mathrm{A}_{2}\right)$ and the modulus of uniform continuity of $g$, respectively, corresponding to the compact $[0, T] \times B\left[0 ; R^{\prime}\right]$. Moreover for every $\bar{t} \in[0, T[$ one has

$$
|V(t, x)-V(\bar{t}, x)| \leq \eta_{\bar{t}}(|t-\bar{t}|)
$$


for every $(t, x) \in\left[0, T\left[\times B[0 ; R]\right.\right.$, where the modulus $\eta_{\bar{t}}$ is defined by

$$
\begin{aligned}
\eta_{\bar{t}}(s)=\max \{ & K_{1} s+K_{2} \omega_{\ell}\left(K_{3} s\right)+\omega_{g}\left(K_{3} s\right), \\
& \left.K_{4} \omega_{\ell}\left(K_{5}\left(\frac{T+1}{T-\bar{t}} s+\omega_{f}(s)\right)\right)+\omega_{g}\left(K_{5}\left(\frac{s}{T-\bar{t}}+\omega_{f}(s)\right)\right)\right\}
\end{aligned}
$$

for suitable positive constants $K_{1}, K_{2}, K_{3}, K_{4}, K_{5}$, depending on $R$, and $\omega_{f}$ is identified with the modulus in $\left(\mathrm{A}_{1}\right)$ corresponding to

$$
Q=[0, T] \times B\left[0 ; R^{\prime}\right]
$$

Proof. Let us recall that $V=V_{e}$ in $\left[0, T\left[\times \mathbb{R}^{n}\right.\right.$, and let us begin by proving that $V_{e}$ is continuous in $x$, uniformly with respect to $t$, for $(t, x) \in[0, T] \times$ $B[0, R]$. By the coercivity condition $\left(\mathrm{A}_{e_{4}}\right)$ and by the obvious local boundedness of $V_{e}$, if the initial conditions are taken in $B[0 ; R]$ then it is not restrictive to consider only those space-time controls $\left(w_{0}, w\right) \in \Gamma(t)$ that satisfy

$$
\int_{0}^{1}\left(w_{0}(s)+|w(s)|\right)^{\beta} d s \leq K_{R}
$$

where $K_{R}$ is a suitable constant depending on $R$. By applying Hölder's inequality one obtains

$$
\int_{0}^{1}\left(w_{0}(s)+|w(s)|\right)^{\alpha} w_{0}(s)^{\beta-\alpha} d s \leq T^{1-\alpha / \beta}\left(K_{R}\right)^{\alpha / \beta} \leq(T+1)\left(K_{R}+1\right) .
$$

Hence, in view of $\left(\mathrm{A}_{e_{3}}\right)$, and Gronwall's Lemma, we can assume that there exists a ball $B\left[0 ; R^{\prime}\right] \subset \mathbb{R}^{n}$ containing all the trajectories issuing from $B[0 ; R]$. Let us fix $\tau \in[0, T], x_{1}, x_{2} \in B[0 ; R], \varepsilon>0$, and let us take a control $\left(w_{0}, w\right) \in \Gamma(\tau)$ such that ((4.4) is verified and)

$$
\int_{0}^{1} \bar{\ell}\left(t_{\left(\tau, x_{2}\right)}(s), x_{\left(\tau, x_{2}\right)}(s), w_{0}(s), w(s)\right) d s+g\left(x_{\left(\tau, x_{2}\right)}(1)\right) \leq V_{e}\left(\tau, x_{2}\right)+\varepsilon,
$$

where $\left(t_{\left(\tau, x_{2}\right)}, x_{\left(\tau, x_{2}\right)}\right)(\cdot) \doteq(t, y)_{\left(\tau, x_{2}\right)}\left[\left(w_{0}, w\right)\right](\cdot)$. Hence, setting

$$
\left(t_{\left(\tau, x_{1}\right)}, x_{\left(\tau, x_{1}\right)}\right)(\cdot) \doteq(t, y)_{\left(\tau, x_{1}\right)}\left[\left(w_{0}, w\right)\right](\cdot),
$$

we have 


$$
\begin{aligned}
& V_{e}\left(\tau, x_{1}\right)-V_{e}\left(\tau, x_{2}\right) \\
& \leq \int_{0}^{1} \bar{\ell}\left(t_{\left(\tau, x_{1}\right)}(s), x_{\left(\tau, x_{1}\right)}(s), w_{0}(s), w(s)\right)-\bar{\ell}\left(t_{\left(\tau, x_{2}\right)}(s), x_{\left(\tau, x_{2}\right)}(s), w_{0}(s), w(s)\right) d s \\
& \quad+g\left(x_{\left(\tau, x_{1}\right)}(1)\right)-g\left(x_{\left(\tau, x_{2}\right)}(1)\right)+\varepsilon \\
& \leq \int_{0}^{1}\left(w_{0}^{\beta}(s)+|w(s)|^{\beta}\right) \omega_{\ell}\left(\left|x_{\left(\tau, x_{1}\right)}(s)-x_{\left(\tau, x_{2}\right)}(s)\right|\right) d s \\
& \quad+g\left(x_{\left(\tau, x_{1}\right)}(1)\right)-g\left(x_{\left(\tau, x_{2}\right)}(1)\right)+\varepsilon .
\end{aligned}
$$

If $L_{R^{\prime}}$ denotes the determination of $L$ in $\left(\mathrm{A}_{e_{1}}\right)$ when $Q=[0, T] \times B\left[0 ; R^{\prime}\right]$, one has

$$
\left|x_{\left(\tau, x_{1}\right)}(s)-x_{\left(\tau, x_{2}\right)}(s)\right| \leq\left|x_{1}-x_{2}\right| e^{L_{R^{\prime}}(T+1)\left(K_{R}+1\right)} \quad \forall s \in[\tau, T],
$$

which, in turn, yields

$$
\begin{aligned}
V_{e}\left(\tau, x_{1}\right)-V_{e}\left(\tau, x_{2}\right) \leq K_{R} \omega_{\ell}\left(\left|x_{1}-x_{2}\right| e^{L_{R^{\prime}}(T+1)\left(K_{R}+1\right)}\right) \\
+\omega_{g}\left(\left|x_{1}-x_{2}\right| e^{L_{R^{\prime}}(T+1)\left(K_{R}+1\right)}\right)+\varepsilon
\end{aligned}
$$

where $\omega_{g}$ and $\omega_{l}$ are the modulus (in $[0, T] \times B\left[0 ; R^{\prime}\right]$ ) of continuity of $g$ and $\ell$ respectively. Since $\varepsilon$ is arbitrary, by exchanging $\left(\tau, x_{1}\right)$ with $\left(\tau, x_{2}\right)$ we obtain (4.1) with $B_{1} \doteq K_{R}$ and $B_{2} \doteq e^{L_{R^{\prime}}(T+1)\left(K_{R}+1\right)}$.

In order to prove the continuity with respect to $t$, let us fix $\left(t_{1}, x\right)$ and $\left(t_{2}, x\right)$ in $[0, T] \times B[0 ; R]$ and let us suppose $t_{1} \leq t_{2}$. We start estimating $V_{e}\left(t_{1}, x\right)-$ $V_{e}\left(t_{2}, x\right)$, assuming that this difference is nonnegative. Let us set

$$
(\bar{t}, \bar{x})(\cdot) \doteq(t, y)_{\left(t_{1}, x\right)}\left[w_{0}^{*}, 0\right](\cdot),
$$

with $w_{0}^{*}(s) \doteq\left(T-t_{1}\right)^{1 / \beta}$ for all $s \in[0,1]$. By the Dynamic Programming Principle we obtain

$$
V_{e}\left(t_{1}, x\right)-V_{e}\left(t_{2}, x\right) \leq \int_{0}^{s_{2}} \bar{\ell}\left((\bar{t}, \bar{x})(s), w_{0}^{*}, 0\right) d s+V_{e}\left(t_{2}, \bar{x}\left(t_{2}\right)\right)-V_{e}\left(t_{2}, x\right),
$$

where $s_{2} \doteq\left(t_{2}-t_{1}\right)\left(T-t_{1}\right)^{-1}$. If $M \doteq \max \left\{M_{1}+M_{2}, 1\right\}$, by $\left(\mathrm{A}_{e_{3}}\right)$ we have

$$
\left|\bar{x}\left(t_{2}\right)-x\right|=\left|\bar{x}\left(t_{2}\right)-\bar{x}\left(t_{1}\right)\right| \leq M\left(1+R^{\prime}\right)\left|t_{1}-t_{2}\right|
$$

and, setting $K_{R}^{\prime} \doteq \max _{(t, x) \in[0, T] \times B\left[0 ; R^{\prime}\right]} \bar{\ell}(t, x, 1,0)$, by the homogeneity of $\bar{\ell}$ (see (ii) 
of Proposition 2.1) and by the first part of the proof we obtain

$$
\begin{aligned}
0 \leq & V_{e}\left(t_{1}, x\right)-V_{e}\left(t_{2}, x\right) \\
\leq & K_{R}^{\prime}\left|t_{2}-t_{1}\right|+K_{R} \omega_{\ell}\left(M\left(1+R^{\prime}\right)\left|t_{2}-t_{1}\right| e^{L_{R^{\prime}}(T+1)\left(K_{R}+1\right)}\right) \\
& +\omega_{g}\left(M\left(1+R^{\prime}\right)\left|t_{2}-t_{1}\right| e^{L_{R^{\prime}}(T+1)\left(K_{R}+1\right)}\right) .
\end{aligned}
$$

Still in the case $t_{1} \leq t_{2}(<T)$, let us estimate the difference $V\left(t_{2}, x\right)-V\left(t_{1}, x\right)$, which we assume nonnegative (otherwise we are in the previous case). Let $\varepsilon>0$ and $c_{1} \in C\left(t_{1}\right)$ be a control so that if we denote by $\tilde{x}(\cdot) \doteq x_{\left(t_{1}, x\right)}\left[c_{1}\right](\cdot)$, we have

$$
V\left(t_{1}, x\right) \geq \int_{t_{1}}^{T} \ell\left(s, \tilde{x}(s), c_{1}(s)\right) d s+g(\tilde{x}(T))-\varepsilon .
$$

Let us set $r \doteq\left(T-t_{2}\right) /\left(T-t_{1}\right)$ and let us define the control $c_{2} \doteq c_{1} \circ J^{-1}$, where $J:\left[t_{1}, T\right] \rightarrow\left[t_{2}, T\right]$ is defined by $J(\tau) \doteq t_{2}+r\left(\tau-t_{1}\right)$. Then $c_{2} \in C\left(t_{2}\right)$, and setting

$$
\stackrel{\vee}{x}(\cdot) \doteq x_{\left(t_{2}, x\right)}\left[c_{2}\right](\cdot),
$$

we have:

$$
|\check{x} \circ J(\tau)-\tilde{x}(\tau)| \leq \int_{t_{1}}^{\tau}\left|r f\left(J(s), \stackrel{v}{x} \circ J(s), c_{2} \circ J(s)\right)-f\left(s, \tilde{x}(s), c_{1}(s)\right)\right| d s
$$

for every $\tau \in\left[t_{1}, T\right]$. Hence, by adding and subtracting $f\left(J(s), \ddot{x} \circ J(s), c_{2} \circ\right.$ $J(s)$ ) inside the last integral, we obtain:

$$
\begin{aligned}
& |\stackrel{v}{x} \circ J(\tau)-\tilde{x}(\tau)| \\
& \leq \frac{\left|t_{1}-t_{2}\right|}{T-t_{1}} \int_{t_{1}}^{T}\left|f\left(J(s), \stackrel{v}{x} \circ J(s), c_{2} \circ J(s)\right)\right| d s \\
& +\int_{t_{1}}^{\tau}\left|f\left(J(s), \stackrel{\vee}{x} \circ J(s), c_{1}(s)\right)-f\left(s, \tilde{x}(s), c_{1}(s)\right)\right| d s \\
& \leq A_{R} \frac{\left|t_{1}-t_{2}\right|}{T-t_{1}}+\int_{t_{1}}^{\tau}\left(1+\left|c_{1}(s)\right|^{\alpha}\right)\left(L_{R^{\prime}}\left|\tilde{x}^{\vee} \circ J(s)-\tilde{x}(s)\right|+\omega_{f}(|J(s)-s|)\right) d s,
\end{aligned}
$$

for every $\tau \in\left[t_{1}, T\right]$ and where we have set

$$
A_{R} \doteq M\left(1+R^{\prime}\right)(T+1)\left(K_{R}+1\right)
$$

Notice that $|J(s)-s| \leq\left|t_{2}-t_{1}\right|$ for every $s \in\left[t_{1}, T\right]$. Hence, by Gronwall's 
Lemma we obtain

$$
|\grave{x} \circ J(\tau)-\tilde{x}(\tau)| \leq e^{L_{R^{\prime}}(T+1)\left(K_{R}+1\right)} A_{R}\left[\frac{\left|t_{1}-t_{2}\right|}{T-t_{1}}+\omega_{f}\left(\left|t_{2}-t_{1}\right|\right)\right]
$$

for every $\tau \in\left[t_{1}, T\right]$. By

$$
\begin{aligned}
V\left(t_{2}, x\right)-V\left(t_{1}, x\right) \leq & \int_{t_{2}}^{T} \ell\left(s, \stackrel{v}{x}(s), c_{2}(s)\right) d s \\
& \quad-\int_{t_{1}}^{T} \ell\left(s, \tilde{x}(s), c_{1}(s)\right) d s+g(\check{x}(T))-g(\tilde{x}(T))+\varepsilon
\end{aligned}
$$

and (4.3), one obtains

$$
\begin{aligned}
V\left(t_{2}, x\right)- & V\left(t_{1}, x\right) \\
\leq & \int_{t_{1}}^{T}\left|\ell\left(J(s), \grave{x} \circ J(s), c_{2} \circ J(s)\right)-\ell\left(s, \tilde{x}(s), c_{1}(s)\right)\right| d s \\
& +\omega_{g}\left(e^{L_{R^{\prime}}(T+1)\left(K_{R}+1\right)} A_{R}\left[\frac{\left|t_{1}-t_{2}\right|}{T-t_{1}}+\omega_{f}\left(\left|t_{2}-t_{1}\right|\right)\right]\right)+\varepsilon \\
\leq & \int_{t_{1}}^{T}\left(1+\left|c_{1}(s)\right|^{\beta}\right) \omega_{\ell}(|(J(s), \dot{x} \circ J(s))-(s, \tilde{x}(s))|) d s \\
& +\omega_{g}\left(e^{L_{R^{\prime}}(T+1)\left(K_{R}+1\right)} A_{R}\left[\frac{\left|t_{1}-t_{2}\right|}{T-t_{1}}+\omega_{f}\left(\left|t_{2}-t_{1}\right|\right)\right]\right)+\varepsilon \\
\leq & K_{R} \omega_{\ell}\left(e^{L_{R^{\prime}}(T+1)\left(K_{R}+1\right)} A_{R}\left[\frac{T+1}{T-t_{1}}\left|t_{1}-t_{2}\right|+\omega_{f}\left(\left|t_{2}-t_{1}\right|\right)\right]\right) \\
& +\omega_{g}\left(e^{L_{R^{\prime}}(T+1)\left(K_{R}+1\right)} A_{R}\left[\frac{\left|t_{1}-t_{2}\right|}{T-t_{1}}+\omega_{f}\left(\left|t_{2}-t_{1}\right|\right)\right]\right)+\varepsilon .
\end{aligned}
$$

By interchanging the roles of $t_{1}$ and $t_{2}$ and observing that $\left(t_{1}-t_{2}\right) /\left(T-t_{2}\right) \leq$ $\left(t_{1}-t_{2}\right) /\left(T-t_{1}\right)$ whenever $t_{2} \leq t_{1}$, by (4.6) and (4.7), one obtains (4.2) with

$$
\begin{aligned}
& \eta_{\bar{t}}(s) \doteq \max \left\{K_{R}^{\prime} s+\right.\left(K_{R} \omega_{\ell}+\omega_{g}\right)\left(M\left(1+R^{\prime}\right) e^{L_{R^{\prime}}(T+1)\left(K_{R}+1\right)} s\right) \\
& K_{R} \omega_{\ell}\left(e^{L_{R^{\prime}}(T+1)\left(K_{R}+1\right)} A_{R}\left[\frac{T+1}{T-\bar{t}} s+\omega_{f}(s)\right]\right) \\
&\left.+\omega_{g}\left(e^{L_{R^{\prime}}(T+1)\left(K_{R}+1\right)} A_{R}\left[\frac{s}{T-\bar{t}}+\omega_{f}(s)\right]\right)\right\} .
\end{aligned}
$$

Let us notice that there is a loss of regularity in the $t$-dependence of $V$ when $t$ approaches $T$. However, as soon as $\alpha<\beta$ and $\ell$ and $g$ are Hölder continuous 
in $(t, x)$, this drawback can be overcome, as it is shown in Theorem 4.2 below (which extends a former result by M. Bardi and F. Da Lio [3]). Finally, if $\ell$ and $g$ are locally Lipschitz continuous in $(t, x)$ and a suitable quantitative condition is verified (see $(\mathrm{NJ})$ condition below), the same improvement can be achieved also for $\alpha=\beta$ (Theorem 4.3). In fact, let us specialize $\left(\mathrm{A}_{2}\right)$ and $\left(\mathrm{A}_{5}\right)$ into the following hypotheses $\left(\mathrm{A}_{2}^{\#}\right)$ and $\left(\mathrm{A}_{5}^{\#}\right)$, respectively:

There exists $\gamma \in] 0,1]$ such that, for every compact $Q \subset[0, T] \times \mathbb{R}^{n}$ there are constants $K_{\ell}$ and $K_{\mathfrak{g}}$ verifying

$\left(\mathrm{A}_{2}^{\#}\right) \quad\left|\ell\left(t_{1}, x_{1}, c\right)-\ell\left(t_{2}, x_{2}, c\right)\right| \leq\left(1+|c|^{\beta}\right) K_{\ell}\left|\left(t_{1}, x_{1}\right)-\left(t_{2}, x_{2}\right)\right|^{\gamma}$

and

$\left(\mathrm{A}_{5}^{\sharp}\right) \quad\left|g\left(x_{1}\right)-g\left(x_{2}\right)\right| \leq K_{g}\left|x_{1}-x_{2}\right|^{\gamma} \quad \forall\left(t_{1}, x_{1}\right)\left(t_{2}, x_{2}\right) \in Q$.

In order to state the next two theorems we need to define some constants. For every $R>0$ choose $R^{\prime} \geq R, K_{R}$ and $L_{R^{\prime}}$ as in the beginning of the proof of Theorem 4.1 , and let $K_{\ell}, K_{g}$ be the determinations of the constants appearing in $\left(\mathrm{A}_{2}^{\#}\right),\left(\mathrm{A}_{5}^{\#}\right)$, respectively, when $Q=[0, T] \times B\left[0, R^{\prime}\right]$. Finally let $M_{1}, M_{2}, \ell_{0}$ be as in $\left(\mathrm{A}_{3}\right)$ and $\left(\mathrm{A}_{4}\right)$. Let us set

$$
M_{R} \doteq\left(K_{R} K_{\ell}+K_{g}\right) e^{L_{R^{\prime}}(T+1)\left(K_{R}+1\right)}
$$

and

$$
G_{R} \doteq e^{L_{R^{\prime}}(T+1)\left(K_{R}+1\right)}\left(K_{R} K_{\ell}+K_{\mathfrak{g}}\right)\left(M_{1}\left(1+R^{\prime}\right)+M_{2}\right) .
$$

Theorem 4.2. Suppose $\alpha<\beta$, assume $\left(\mathrm{A}_{1}\right),\left(\mathrm{A}_{3}\right),\left(\mathrm{A}_{2}^{\#}\right),\left(\mathrm{A}_{4}\right)$, and $\left(\mathrm{A}_{5}^{\sharp}\right)$, and fix $R>0$. Then

$$
\left|V\left(t, x_{1}\right)-V\left(t, x_{2}\right)\right| \leq M_{R}\left|x_{1}-x_{2}\right|^{\gamma}
$$

for every $\left(t, x_{1}\right),\left(t, x_{2}\right) \in[0, T] \times B[0 ; R]$. Moreover, there exists a constant $\bar{K}_{R}$ such that

$$
\left|V\left(t_{1}, x\right)-V\left(t_{2}, x\right)\right| \leq \bar{K}_{R}\left|t_{1}-t_{2}\right|^{\gamma(\beta-\alpha) /(\beta-\gamma \alpha)}
$$

for every $\left(t_{1}, x\right)\left(t_{2}, x\right) \in[0, T] \times B[0 ; R]$.

Finally if in $\left(\mathrm{A}_{1}\right)$ one has $\omega_{f}(s) \doteq L_{f} s$ for some $L_{f}>0$, the map $t \rightarrow V(t, x)$ is locally $\gamma$-Hölder continuous on $[0, T]$ uniformly with respect to $x \in B[0 ; R]$.

Theorem 4.3. Suppose $\alpha=\beta$ and assume $\left(\mathrm{A}_{1}\right),\left(\mathrm{A}_{3}\right),\left(\mathrm{A}_{2}^{\#}\right),\left(\mathrm{A}_{4}\right)$, and $\left(\mathrm{A}_{5}^{\#}\right)$, 
with $\gamma=1$. Fix $R>0$ and assume that the following no-jump condition

$$
G_{R} \leq \ell_{0}
$$

is verified. Then there exists a constant $B_{3}$ depending on $R$ such that

$$
\left|V\left(t_{1}, x_{1}\right)-V\left(t_{2}, x_{2}\right)\right| \leq B_{3}\left|\left(t_{1}, x_{1}\right)-\left(t_{2}, x_{2}\right)\right|
$$

for every $\left(t_{1}, x_{1}\right)\left(t_{2}, x_{2}\right) \in B[0 ; R]$.

Proof of Theorem 4.2. The estimate (4.8) is equivalent to (4.1) for $\omega_{\ell}(\cdot)=$ $K_{\ell}|(\cdot)|^{\gamma}$ and $\omega_{g}(\cdot)=K_{g}|(\cdot)|^{\gamma}$. Moreover, the statement concerning the local $\gamma$-Hölder continuity of $t \rightarrow V(t, x)$ is a straightforward consequence of (4.2). In order to prove (4.9), let us observe that in view of the proof of Theorem 4.1 it is sufficient to consider the case when $t_{1} \leq t_{2}$ and $V\left(t_{2}, x\right) \leq V\left(t_{1}, x\right)$, the other case (that is $V\left(t_{1}, x\right) \leq V\left(t_{2}, x\right)$ ) being proven with estimate (4.6). By the Dynamic Programming Principle for every pair $t_{1}, t_{2} \in[0, T]$ such that $t_{1} \leq t_{2}$, there exists a control $c_{t_{1} t_{2}} \in C\left(t_{1}\right)$ such that setting $\tilde{x} \doteq x_{\left(t_{1}, x\right)}\left[c_{t_{1} t_{2}}\right]$, we have

$$
\begin{aligned}
0 & \leq V\left(t_{2}, x\right)-V\left(t_{1}, x\right) \\
& \leq V\left(t_{2}, x\right)-V\left(t_{2}, \tilde{x}\left(t_{2}\right)\right)-\int_{t_{1}}^{t_{2}} \ell\left(s, \tilde{x}(s), c_{t_{1} t_{2}}(s)\right) d s+\left|t_{1}-t_{2}\right| .
\end{aligned}
$$

Hence by (4.8), the coercivity assumption $\left(A_{4}\right)$ and the growth condition $\left(A_{3}\right)$ one obtains

$$
\begin{aligned}
0 \leq & V\left(t_{2}, x\right)-V\left(t_{1}, x\right) \\
\leq & \left(K_{R} K_{\ell}+K_{g}\right)\left|x-\tilde{x}\left(t_{2}\right)\right|^{\gamma} e^{L_{R^{\prime}}(T+1)\left(K_{R}+1\right)} \\
& \quad-\int_{t_{1}}^{t_{2}} \ell_{0}\left|c_{t_{1} t_{2}}(s)\right|^{\beta} d s+\left(\ell_{1}+1\right)\left|t_{1}-t_{2}\right| \\
\leq & e^{L_{R^{\prime}}(T+1)\left(K_{R}+1\right)}\left(K_{R} K_{\ell}+K_{g}\right)\left(M_{1}\left(1+R^{\prime}\right)+M_{2}\right)^{\gamma}\left(\int_{t_{1}}^{t_{2}}\left(1+\left|c_{t_{1} t_{2}}(s)\right|^{\alpha}\right) d s\right)^{\gamma} \\
& \quad-\int_{t_{1}}^{t_{2}} \ell_{0}\left|c_{t_{1} t_{2}}(s)\right|^{\beta} d s+\left(\ell_{1}+1\right)\left|t_{1}-t_{2}\right| .
\end{aligned}
$$

By Hölder's inequality we have

$$
\int_{t_{1}}^{t_{2}}\left|c_{t_{1} t_{2}}(s)\right|^{\alpha} d s \leq\left(\int_{t_{1}}^{t_{2}}\left|c_{t_{1} t_{2}}(s)\right|^{\beta} d s\right)^{\alpha / \beta}\left|t_{1}-t_{2}\right|^{1-\alpha / \beta} .
$$


Moreover, we claim that there exists $K^{\prime}>0$ such that

$$
\int_{t_{1}}^{t_{2}}\left|c_{t_{1} t_{2}}(s)\right|^{\beta} d s \leq K^{\prime}\left|t_{1}-t_{2}\right|^{s}
$$

where $s=\gamma(\beta-\alpha) /(\beta-\gamma \alpha)$. Indeed, suppose by contradiction that

$$
\sup _{\substack{t_{1}, t_{2} \in[0, T] \\ t_{1}<t_{2}}} \frac{\int_{t_{1}}^{t_{2}}\left|c_{t_{1}} t_{2}(s)\right|^{\beta} d s}{\left|t_{1}-t_{2}\right|^{s}}=+\infty .
$$

Hence there exists a sequence of pairs $\left(t_{1_{n}}, t_{2_{n}}\right) \in[0, T]^{2}$ such that $t_{1_{n}} \leq t_{2_{n}}$ and

$$
\frac{\int_{t_{1 n}}^{t_{2 n}}\left|c_{t_{1} t_{2}}(s)\right|^{\beta} d s}{\left|t_{2 n}-t_{1_{n}}\right|^{s}}>n .
$$

Plugging (4.11) in (4.10), one obtains

$$
\begin{aligned}
& 0 \leq \frac{V\left(t_{2}, x\right)-V\left(t_{1}, x\right)}{\left|t_{2}-t_{1}\right|^{s}} \\
& \leq \widetilde{K} \frac{\left(\int_{t_{1}}^{t_{2}}\left|c_{t_{1} t_{2}}(s)\right|^{\beta}\right)^{\gamma(\alpha / \beta)}\left|t_{2}-t_{1}\right|^{\gamma(1-\alpha / \beta)}}{\left|t_{2}-t_{1}\right|^{s}}-\ell_{0} \frac{\int_{t_{1}}^{t_{2}}\left|c_{t_{1} t_{2}}(s)\right|^{\beta} d s}{\left|t_{2}-t_{1}\right|^{s}} \\
&+\frac{\left(\ell_{1}+1\right)}{\left|t_{1}-t_{2}\right|^{s-1}}
\end{aligned}
$$

for a suitable $\tilde{K}$. For every $n \in \mathbb{N}$ let us set $t_{1}=t_{1_{n}}$ and $t_{2}=t_{2_{n}}$. It is easy to check that the right hand side of (4.12) approaches $-\infty$ as $n$ tends to infinity, which gives a contradiction. Therefore there exists $K^{\prime}$ such that

$$
\int_{t_{1}}^{t_{2}}\left|c_{t_{1} t_{2}}(s)\right|^{\beta} d s \leq K^{\prime}\left|t_{2}-t_{1}\right|^{\gamma(\beta-\alpha) /(\beta-\gamma \alpha)}
$$

Plugging this last estimate in (4.10) we obtain (4.9), for a suitable constant $\bar{K}_{R}$.

Proof of Theorem 4.3. In view of the proof of Theorem 4.1 we have to prove 
only that there exists a constant $B_{3}$ such that

$$
V\left(t_{2}, x\right)-V\left(t_{1}, x\right) \leq B_{3}\left|\left(t_{1}, x_{1}\right)-\left(t_{2}, x_{2}\right)\right|,
$$

supposing $t_{1} \leq t_{2}$ and $V\left(t_{2}, x\right)-V\left(t_{1}, x\right) \geq 0$. Let us rewrite inequality (4.10) for the special case when $\gamma=1$ and $\alpha=\beta$ :

$$
\begin{aligned}
0 & \leq V\left(t_{2}, x\right)-V\left(t_{1}, x\right) \\
& \leq e^{L_{R^{\prime}}(T+1)\left(K_{R}+1\right)}\left(K_{R} K_{\ell}+K_{g}\right)\left(M_{1}\left(1+R^{\prime}\right)+M_{2}\right) \\
& \quad \int_{t_{1}}^{t_{2}}\left(1+\left|c_{t_{1} t_{2}}(s)\right|^{\beta}\right) d s-\int_{t_{1}}^{t_{2}} \ell_{0}\left|c_{t_{1} t_{2}}(s)\right|^{\beta} d s+\left(l_{1}+1\right)\left|t_{1}-t_{2}\right| .
\end{aligned}
$$

We claim that there exists a constant $K^{\prime}$ such that

$$
\int_{t_{1}}^{t_{2}}\left|c_{t_{1} t_{2}}(s)\right|^{\beta} d s \leq K^{\prime}\left|t_{1}-t_{2}\right|
$$

Assume by contradiction that this is false and argue as in the proof of Theorem 4.2 to obtain

$$
\begin{aligned}
0 & \leq \frac{V\left(t_{2}, x\right)-V\left(t_{1}, x\right)}{\left|t_{2}-t_{1}\right|} \\
& \leq G_{R} \frac{\int_{t_{1}}^{t_{2}}\left|c_{t_{1} t_{2}}(s)\right|^{\beta} d s}{\left|t_{2}-t_{1}\right|}-l_{0} \frac{\int_{t_{1}}^{t_{2}}\left|c_{t_{1} t_{2}}(s)\right|^{\beta}}{\left|t_{2}-t_{1}\right|}+\tilde{\ell},
\end{aligned}
$$

where $\tilde{\ell}$ is a suitable positive constant. In view of condition $(\mathrm{NJ})$ the right-hand side of (4.13) tends to $-\infty$, as $n$ goes to infinity, a contradiction. Plugging (4.14) in (4.13), we can finish.

\section{APPENDIX}

Removing the hypothesis that $C$ is a cone. For the sake of simplicity the unbounded control set $C$ has been assumed to be a closed cone. However, all the results of the present paper can be generalized to the case when $C$ is any closed subset of $\mathbb{R}^{m}$. We sketch briefly the changes that are needed in order to address the general case. Define the conic hull Con $(E)$ of a set $E \subseteq \mathbb{R}^{q}$ by setting

$$
\operatorname{Con}(E) \doteq\{\lambda e, \lambda \geq 0, e \in E\} .
$$


If one redefines the Hamiltonian $H_{e}$ appearing in the equation $\left(\mathrm{HJ}_{e}\right)$ by setting

$$
H_{e} \doteq \sup _{\left(w_{0}, w\right) \in \operatorname{Con}(\{1\} \times C) \cap S_{+}^{m}}\left\{-p_{0} w_{0}^{\beta}-\left\langle p, \bar{f}\left(t, x, w_{0}, w\right)\right\rangle-\bar{\ell}\left(t, x, w_{0}, w\right)\right\}
$$

then in Section 2 everything works for a general set $C$, up to the obvious replacement of the set $[0,+\infty[\times C$ with $\overline{\operatorname{Con}(\{1\} \times C)}$. (Incidentally, notice that, as soon as $C$ is a closed cone, one has $[0,+\infty[\times C=\overline{\operatorname{Con}(\{1\} \times C)}$. In order to generalize Section 3 to the case when $C$ is not a cone, let us redefine the sets $\Gamma^{+}(\bar{t})$ and $\Gamma(\bar{t})$ by setting

$$
\begin{aligned}
\Gamma^{+}(\bar{t}) & \doteq\left\{\left(w_{0}, w\right) \in \mathcal{B}\left([0,1], \operatorname{Con}(\{1\} \times C) \quad \text { such that } \bar{t}+\int_{0}^{1} w_{0}^{\beta}(s) d s=T\right\}\right. \\
\Gamma(\bar{t}) & \doteq\left\{\left(w_{0}, w\right) \in \mathcal{B}([0,1], \overline{\operatorname{Con}(\{1\} \times C)}) \quad \text { such that } \bar{t}+\int_{0}^{1} w_{0}^{\beta}(s) d s=T\right\} .
\end{aligned}
$$

With this position the embedding result stated in Proposition 3.1 is still valid, with an unchanged proof. Proposition 3.2 , which establishes the density of the original trajectories into space-time trajectories, maintains its validity as well. However, when $C$ is not a cone, the proof needs substantial changes. Indeed we are not allowed to use the controls $\left(w_{0_{n}}, w\right)$ introduced in that proof, for in general they do not take values in $\overline{\operatorname{Con}(\{1\} \times C)}$. Briefly, one can proceed in the following way. To begin with, for every $n \in \mathbb{N}$ and every $\left(w_{0}, w\right) \in \overline{\operatorname{Con}(\{1\} \times C)}$ one defines

$$
\Gamma^{n}\left(w_{0}, w\right) \doteq B\left[\left(w_{0}, w\right), \frac{1}{n}\right] \cap\left\{\left(\widetilde{w}_{0}, w\right):\left|w_{0}-\widetilde{w}_{0}\right| \leq \frac{1}{n}\right\}
$$

and

$$
m_{n}\left(w_{0}, w\right) \doteq \max \left\{\widetilde{w}_{0} \quad \text { such that }\left(\widetilde{w}_{0}, \widetilde{w}\right) \in \Gamma^{n}\left(w_{0}, w\right)\right\}
$$

Then one replaces the approximating controls in Proposition 3.2 with the controls $\left(\bar{w}_{0_{n}}, \bar{w}_{n}\right) \doteq \lambda_{n}\left(w_{0_{n}}, w_{n}\right)$ where

$$
\left(w_{0_{n}}, w_{n}\right)(s) \doteq \begin{cases}\left(w_{0}, w\right)(s) & \text { if } s \in[0,1] \backslash w_{0}^{-1}(0), \\ \left(m_{n}\left(w_{0}(s), w(s)\right), w(s)\right) & \text { if } s \in w_{0}^{-1}(0)\end{cases}
$$

and

$$
\lambda_{n} \doteq\left(\frac{T-\bar{t}}{\int_{0}^{1}\left(w_{0_{n}}(s)\right)^{\beta} d s}\right)^{1 / \beta} .
$$


Then standard arguments from set valued analysis imply that $\left(\bar{w}_{0_{n}}, \bar{w}_{n}\right)$ approaches $\left(w_{0}, w\right)$ uniformly, so that the proof of Proposition 3.2 proceeds as in the case when $C$ is not a cone. In view of the above extension, also the remaining part of Section 3 and the whole Section 4 can be generalized to the case where $C$ is not a cone.

\section{REFERENCES}

[1] M. Arisawa And A. Tourin, Regularizing effects for a class of first-order Hamilton-Jacobi equations, Nonlinear Anal. 29 (1997), 1405-1420.

[2] J. P. Aubin and A. Cellina, Differential Inclusion, Springer, Berlin, 1984.

[3] M. BARDi AND F. DA Lio, On the Bellman equation for some unbounded control problems, NoDEA - Nonlinear Differential Equations and Applications 4 (1997), 491-510.

[4] M. BARdi AND P. Soravia, A comparison result for Hamilton-Jacobi equations and applications to some differential games lacking controllability, Funkcial. Ekvac. 37 (1994), 19-43.

[5] G. BARLEs, Regularity results for first-order Hamilton-Jacobi equations, Differential Integral Equations 3 (1990), 103-125.

[6] G. BARLES, An approach of deterministic control problems with unbounded data, Ann. Inst. Henri Poincar 7-4 (1990), 235-258.

[7] G. BARLES, Uniqueness and regularity results for first-order Hamilton-Jacobi equations, Indiana Univ. Math. J. 39 (1990), 443-466.

[8] E.N.Barron, R.Jensen and J.L. Menaldi, Optimal control and differential games with measures, Nonlinear Analysis, Theory, Methods \& Applications 21-4 (1993), 241-268.

[9] A. Bensoussan, Perturbations methods in optimal control, John Wiley and Sons, New York, 1988.

[10] F. Camilli and M. Falcone, Approximation of control problems involving ordinary and impulsive controls, Preprint (1995).

[11] P. Cannarsa and G. Da Prato, Nonlinear optimal control with infinite horizon for distributed parameter systems and stationary Hamilton-Jacobi equations, SIAM J. Control Optim. 27 (1989), 861-875.

[12] M. G. Crandall, L. C. Evans And P. L. Lions, Some properties of viscosity solutions of Hamilton-Jacobi equations, Trans. Amer. Math. Soc. 282 (1984), 487-502..

[13] M. G. Crandall and P. L. Lions, Viscosity solutions of Hamilton-Jacobi equations, Trans. Amer. Math. Soc. 277 (1983), 1-42.

[14] M. G. Crandall And R. Newcomb, Viscosity solutions of Hamilton-Jacobi equations at the boundary, Proc. Amer. Math. Soc. 94 (1985), 283-290.

[15] W. Fleming And H.M. Soner, Controlled Markov processes and viscosity solutions, Springer Verlag, New York, 1993.

[16] H. IsHII, A boundary value problem of the Dirichlet type for Hamilton-Jacobi equations, Ann. Sc. Norm. Sup. Pisa (IV) 16 (1989), 105-135.

[17] H. IsHII, A comparison result for Hamilton-Jacobi equations without growth condition on solutions from above, Appl.Anal. 67 (1997), 357-372.

[18] P. L. Lions, Regularizing effects for first-order Hamilton-Jacobi equations, Applicable Anal. 20 (1985), 283-307.

[19] W. M. McEnEANEy, Uniqueness for viscosity solutions of nonstationary HJB equations under some a priori conditions (with applications), SIAM. J. Control Optim 33 (1995), $1560-1576$.

[20] M. Motta And F. Rampazzo, Nonlinear systems with unbounded controls and state constraints: a problem of proper extension, NoDEA - Nonlinear Differential Equations and Applications 3 (1996), 191-216. 
[21] M.Motta AND F.RAmpazzo, The value function of a slow growth control problem with state constraints, Jour. of Mathematical Systems, Estimation and Control 7,3 (1997), $375-378$.

[22] F. RAmpazzo, Continuity of the upper and lower value of slow growth differential games, J. Math. Anal. Appl. 213 (1997), 15-31.

[23] F. Rampazzo, Differential games with unbounded versus bounded controls, SIAM J. Control Optim. 3 (1998), 814-839.

[24] F. Rampazzo and C. SaRtori, The minimum time function with unbounded controls, Jour. of Mathematical Systems, Estimation and Control 8 (1998), 1-34.

[25] P. Soravia, Optimality principles and representation formulas for viscosity solutions of Hamilton-Jacobi equations. I. Equations of unbounded and degenerate control problems without uniqueness., Adv. Differential Equations 4 (1999), 275-296.

Both authors were partially supported by European Union T.M.R. Program Viscosity Solutions and their applications.

FRANCO RAMPAZZO

Dipartimento di Matematica Pura e Applicata

Universit di Padova

Via Belzoni 7

35131 Padova, Italy

EMAIL: rampazzo@math.unipd.it

C. SARTORI

Dipartimento di Metodi e Modelli Matematici per le Scienze Applicate

Universit di Padova

Via Belzoni 7

35131 Padova, Italy

EMAIL: sartori@dmsa.unipd.it

SUbJECT Classification: 35B37, 49L25,49N25,35D10.

KEYWORDS: Hamiltonians fast growing in the adjoint variable, uniqueness, regularity, unbounded controls.

Submitted: January 20th, 1999, revised: December 2nd, 1999. 